\title{
Counting Morse functions on the 2-sphere
}

\author{
Liviu I. Nicolaescu
}

\begin{abstract}
We count how many 'different' Morse functions exist on the 2-sphere. There are several ways of declaring that two Morse functions $f$ and $g$ are 'indistinguishable' but we concentrate only on two natural equivalence relations: homological (when the regular sublevel sets $f$ and $g$ have identical Betti numbers) and geometric (when $f$ is obtained from $g$ via global, orientation-preserving changes of coordinates on $S^{2}$ and $\mathbb{R}$ ). The count of homological classes is reduced to a count of lattice paths confined to the first quadrant. The count of geometric classes is reduced to a count of certain labeled trees, which is encoded by certain elliptic integrals.
\end{abstract}

\section{The main problem}

Suppose that $X$ is a smooth compact, oriented manifold without boundary. Following Thom, we say that a smooth function $f: X \rightarrow \mathbb{R}$ is an excellent Morse function if all of its critical points are nondegenerate, and no two of them lie on the same level set. We denote by $\mathcal{M}_{X}$ the space of excellent Morse functions on $X$. In the remainder of this introduction a Morse function will by default be excellent.

For $f \in \mathcal{M}_{X}$ we denote by $\nu(f)$ the number of critical points of $f$. Given a Morse function $f \in \mathcal{M}_{X}$ with $\nu(f)=n$ we define a slicing of $f$ to be an increasing sequence of real numbers

$$
a_{0}<a_{1}<\cdots<a_{n-1}<a_{n},
$$

such that, for every $i=1, \ldots, n$ the interval $\left(a_{i-1}, a_{i}\right)$ contains precisely one critical value of $f$.

Two Morse functions $f, g: X \rightarrow \mathbb{R}$ will be called geometrically ${ }^{1}$ equivalent if there exists an orientation-preserving diffeomorphism $R: X \rightarrow X$ and an orientation-preserving diffeomorphism $L: \mathbb{R} \rightarrow \mathbb{R}$ such that

$$
g=L \circ f \circ R^{-1} .
$$

We denote this equivalence relation by $\sim_{g}$.

Two Morse functions $f, g: X \rightarrow \mathbb{R}$ will be called topologically equivalent if $\nu(f)=\nu(g)=n$, and there exists a slicing $a_{0}<a_{1}<\cdots<a_{n}$ of $f$, a slicing $b_{0}<b_{1}<\cdots<b_{n}$ of $g$, and orientationpreserving diffeomorphisms

$$
\phi_{i}:\left\{f \leqslant a_{i}\right\} \rightarrow\left\{g \leqslant b_{i}\right\}, \quad \text { for all } i=1, \ldots, n .
$$

Two Morse functions $f, g: X \rightarrow \mathbb{R}$ will be called homologically equivalent if $\nu(f)=\nu(g)=n$, and there exists a slicing $a_{0}<a_{1}<\cdots<a_{n}$ of $f$, and a slicing $b_{0}<b_{1}<\cdots<b_{n}$ of $g$ such that, for

Received 10 April 2007, accepted in final form 13 April 2008.

2000 Mathematics Subject Classification 05A15, 05A17, 57M15, 58E05.

Keywords: Morse functions, equivalence of smooth functions, Reeb graphs, lattice walks, Catalan numbers, Young lattice, first-order quasilinear partial differential equations, elliptic functions.

This journal is (c) Foundation Compositio Mathematica 2008.

${ }^{1}$ There does not seem to be a consensus amongst geometers on how to refer to what we dubbed geometric equivalence. Our choice of terminology is motivated by the point of view that a geometric property is a property that is independent of the choice of coordinates. 


\section{I. NiCOLAESCU}

every $i=1, \ldots, n$, the sublevel sets $\left\{f \leqslant a_{i}\right\}$ and $\left\{g \leqslant b_{i}\right\}$ have the same Betti numbers. We denote these equivalence relations by $\sim_{t}$ and $\sim_{h}$, respectively. Note that

$$
f_{0} \sim_{g} f_{1} \Longrightarrow f_{0} \sim_{t} f_{1} \Longrightarrow f_{0} \sim_{h} f_{1} .
$$

Set

$$
\mathcal{M}_{X}^{n}:=\left\{f \in \mathcal{M}_{X} ; \nu(f)=n\right\}, \quad\left[\mathcal{M}_{X}^{n}\right]_{*}:=\mathcal{M}_{X}^{n} / \sim_{*}, \quad * \in\{h, t, g\} .
$$

Observe that we have natural projections

$$
\left[\mathcal{M}_{X}^{n}\right]_{g} \rightarrow\left[\mathcal{M}_{X}^{n}\right]_{t} \rightarrow\left[\mathcal{M}_{X}^{n}\right]_{h}
$$

The geometric equivalence classes are open subsets of $\mathcal{M}_{X}^{n}$. This shows that the quotient topology on $\left[\mathcal{M}_{X}^{n}\right]_{*}, * \in\{g, t, h\}$, is discrete.

We can then ask about the combinatoric structures of the sets $\left[\mathcal{M}_{X}^{n}\right]_{*}, * \in\{g, t, h\}$, and it is more realistic ${ }^{2}$ to start by addressing this question for special $X$. In this paper we address this question in the case $X=S^{2}$. We think it is instructive to spend the remainder of this introduction discussing the even simpler case $X=S^{1}$ since it bares some structural similarities with the case $X=S^{2}$ to which we will devote the bulk of the paper.

A Morse function on $S^{1}$ has an even number of critical points. The noncritical sublevel sets of a Morse function on $S^{1}$ are disjoint unions of closed intervals, and we deduce that in this case the topological and homological classifications coincide. Suppose that the Morse function $f: S^{1} \rightarrow \mathbb{R}$ has $2 m+2$ critical points. Fix a slicing $a_{0}<a_{1}<\cdots<a_{2 m+2}$ of $f$, and set

$$
L_{i}:=\left\{f \leqslant a_{i}\right\} .
$$

If we set

$$
x_{i}(f):=b_{0}\left(L_{i}\right)=\text { the number of path components of } L_{i},
$$

then we see that a Morse function defines a sequence

$$
x(f):\{1,2, \ldots, 2 m+1\} \rightarrow \mathbb{Z}_{>0}, \quad i \longmapsto x_{i}(f),
$$

satisfying

$$
x_{1}=x_{2 m+1}=1, \quad x_{i}>0, \quad\left|x_{i+1}-x_{i}\right|=1, \quad \text { for all } i .
$$

We regard such a sequence as a walk of length $2 m$ on the lattice $\mathbb{Z}$ of the horizontal axis $\mathbb{R}$ with steps of size one going East or West, which starts and ends at one, and it is confined to the positive chamber $\mathbb{Z}_{>0}$. (This is a fundamental chamber for the Coxeter group $\mathbb{Z} / 2$.)

Conversely, to any such walk we can associate a Morse function with $2 m+2$ critical points, where a step East corresponds to introducing 0-handle ( $b_{0}$ increases by one), and a step West corresponds to attaching a 1-handle, $b_{0}$ decreases by one. Hence, we have

$$
f \sim_{t} g \Longleftrightarrow x(f)=x(g) .
$$

The number of such confined walks can be easily determined using André's reflection principle, [VW92, Example 14.8], and we obtain

$$
\left[\mathcal{M}_{S^{1}}^{2 m+2}\right]_{h}=\left(\begin{array}{c}
2 m \\
m
\end{array}\right)-\left(\begin{array}{c}
2 m \\
m+2
\end{array}\right)=\frac{1}{m+2}\left(\begin{array}{c}
2 m+2 \\
m+1
\end{array}\right)=C_{m+1},
$$

where $C_{k}=1 /(k+1)\left(\begin{array}{c}2 k \\ k\end{array}\right)$ denotes the $k$ th Catalan number. As we will see, the homology classes of Morse functions on $S^{2}$ is also encoded by lattice paths confined to a fundamental chamber of the Coxeter group $\mathbb{Z} / 2 \times \mathbb{Z} / 2$.

${ }^{2}$ We refer to [Arn07] for a nice discussion of the history and the evolution of this question. 


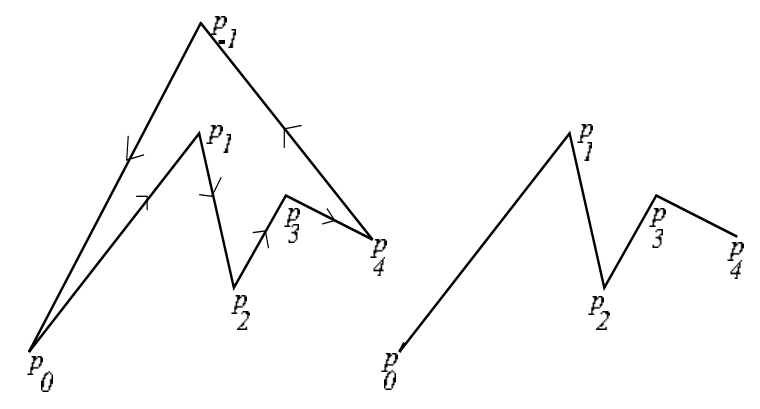

Figure 1. Cutting a Morse function down to a snake.

The number of geometric equivalence classes of Morse functions on $S^{1}$ can be determined using the calculus of snakes of Arnold [Arn91, Arn92]. We outline the main idea.

Suppose that $f: S^{1} \rightarrow \mathbb{R}$ is Morse function with $2 n+2$ critical points. Denote by $p_{-1} \in S^{1}$ the point where $f$ achieves its global maximum. Then, starting at $p_{-1}$ label the critical points $p_{0}, \ldots, p_{2 m}$, in counterclockwise order (see Figure 1 ). Now remove the $\operatorname{arcs}\left[p_{-1}, p_{0}\right),\left(p_{2 m}, p_{-1}\right]$ (see Figure 1). What is left is what Arnold calls an $A_{2 m}$-snake. Their numbers and several associated generating series are determined in [Arn91, Arn92]. One of these generating functions depends on two variables and satisfies a linear first-order partial differential equation (PDE) which can be solved explicitly using the methods of characteristics.

More explicitly, if we denote by $g_{n}$ the number of geometric equivalence classes of Morse functions on $S^{1}$ with $(2 n+2)$ critical points, and we set

$$
g(t)=\sum_{n \geqslant 0} g_{n} \frac{t^{2 n+1}}{(2 n+1) !},
$$

then $g(t)=\tan t$. Equivalently, we can say that $g$ is the inverse of the function

$$
g \mapsto t(g):=\int_{0}^{g}\left(1+s^{2}\right)^{-1} d s .
$$

We will encounter a similar phenomenon when investigating geometric equivalence classes on $S^{2}$, where instead of a rational integral, we will have an elliptic one.

To conclude this introduction, let us give an interpretation from the point of view of singularity theory for the number of geometric equivalence classes of excellent Morse functions on $S^{2}$.

Denote by $\mathcal{F}$ the space of smooth functions on $S^{2}$, and by Diff $+\left(S^{2}\right)$ (respectively Diff $+(\mathbb{R})$ ) the group of orientation-preserving diffeomorphism of $S^{2}$ (respectively $\mathbb{R}$ ). There exists a left action of the 'gauge group' $\mathcal{G}=\operatorname{Diff}_{+}(\mathbb{R}) \times \operatorname{Diff}_{+}\left(S^{2}\right)$ on $\mathcal{F}$ given by

$$
\mathcal{G} \times \mathcal{F} \ni(u, v ; f) \mapsto u \circ f \circ v^{-1} .
$$

Two functions are therefore geometrically equivalent if and only if they belong to the same $\mathcal{G}$-orbit. A function $f \in \mathcal{F}$ is an excellent Morse function if and only if its orbit $\mathcal{G} \cdot f$ is an open subset of $\mathcal{X}$, so that $\mathcal{M}_{S^{2}}$ is precisely the union of the stable orbits.

The complement $\mathcal{S}:=\mathcal{F} \backslash \mathcal{M}_{S^{2}}$ is a codimension-one subvariety. Its smooth locus decomposes into two parts: the Maxwell stratum $\mathcal{S}_{m}$, consisting of Morse functions $f$ which have exactly one pair of critical points on the same level set, and the $A_{2}$-stratum $\mathcal{S}_{a}$, consisting of smooth functions $f$ with have exactly one degenerate critical point $p$, and in a neighborhood of that point one can find local coordinates $(x, y)$ such that

$$
x(p)=y(p)=0, \quad f(x, y)=f(p)+x^{3} \pm y^{2} .
$$




\section{I. NicOlAESCU}

The Maxwell and $A_{2}$ strata are transversally oriented, and they define a chamber structure on $\mathcal{F}$. On the $A_{2}$-stratum we choose the transversal orientation corresponding to the birth process, that is, when crossing the $A_{2}$ stratum from the negative side to the positive side the number of critical points increases by two. Denote by $\mathcal{S}_{a}^{n}$ the part of the $A_{2}$ stratum consisting of functions with $2 n+1$ critical points. Then the number of geometric equivalence classes of excellent Morse functions with $2 n$ critical points is the number of chambers between the strata $\mathcal{S}_{a}^{n-1}$ and $\mathcal{S}_{a}^{n}$.

\section{The main results}

In $\S 3$, using the basics of Morse theory, we analyze what kind of handle additions can occur as we cross the critical levels of a Morse function on $S^{2}$, and we describe a bijection between the set of geometric equivalence classes of Morse functions on $S^{2}$ and the set of Morse trees. These are labelled trees with vertices of degree one or three such that any vertex of degree three has at least one neighbor with a bigger label, and at least one neighbor with a smaller label. This correspondence from Morse functions to labelled graphs first appeared on the mathematical scene in the work of Reeb [Ree46].

In $\S 4$ we describe a bijection between homology classes of Morse functions on $S^{2}$ and certain lattice paths in $\mathbb{Z}_{>0}^{2}$ and we deduce that the number of homology classes of Morse functions on $S^{2}$ with $(2 n+2)$ critical points is $C_{n} C_{n+1}$, where $C_{n}$ denotes the $n$th Catalan number.

In $\S 5$ we produce a two-parameter recurrence for the number of Morse trees (Theorem 5.1) which is computationally very effective. In $\S 6$ we associate to this two-parameter family of numbers an exponential type generating function of two independent variables, and we prove that this function satisfies a first-order quasilinear PDE. The classical method of characteristics [CH89], reduces this equation to a Hamiltonian system in the plane, where the Hamiltonian function is a cubic polynomial. This allows us to obtain a concrete description of the exponential generating function of the number of geometric classes.

More precisely, if $\xi_{n}$ denotes the number of geometric classes of Morse functions with $2 n+2$ critical points and

$$
\xi(t)=\sum_{n \geqslant 0} \xi_{n} \frac{t^{2 n+1}}{(2 n+1) !},
$$

then in Theorem 6.1 we show that $\xi$ is the inverse of the function

$$
\xi \mapsto \theta(\xi)=\int_{0}^{\xi} \frac{d \tau}{\sqrt{\tau^{4} / 4-\tau^{2}+2 \xi \tau+1}} .
$$

From this we can obtain a description of $\xi$ in terms of the classical Weierstrass elliptic functions $\wp$.

The set of topological equivalence classes of Morse functions seems very mysterious at this time. It has resisted all of our attempts to uncover a computationally friendly structure. In $\S 7$ we present a partial result. Using some results on counts of paths in the Young lattice of partitions we were able to produce a lower bound for the number of topology classes of Morse functions. More precisely, we show that there exist at least $1 \cdot 3 \cdots(2 n+1)$ topological equivalence classes of Morse functions on $S^{2}$ with $2 n+2$ critical points.

\section{The anatomy of a Morse function on the 2-sphere}

Denote by $[n]$ the set $\{1,2, \ldots, n\}$. Suppose that $f$ is an excellent Morse function on the 2-sphere. In this case the number of critical points $\nu(f)$ is a positive even integer, we set $\bar{\nu}(f):=\frac{1}{2} \nu(f)-1$, and we say that $\bar{\nu}(f)$ is the order of $f$. From the Morse inequalities we deduce that the order of a Morse function is equal to the number of its saddle points. 
Morse FUnCTIONS ON THE 2-SPHERE

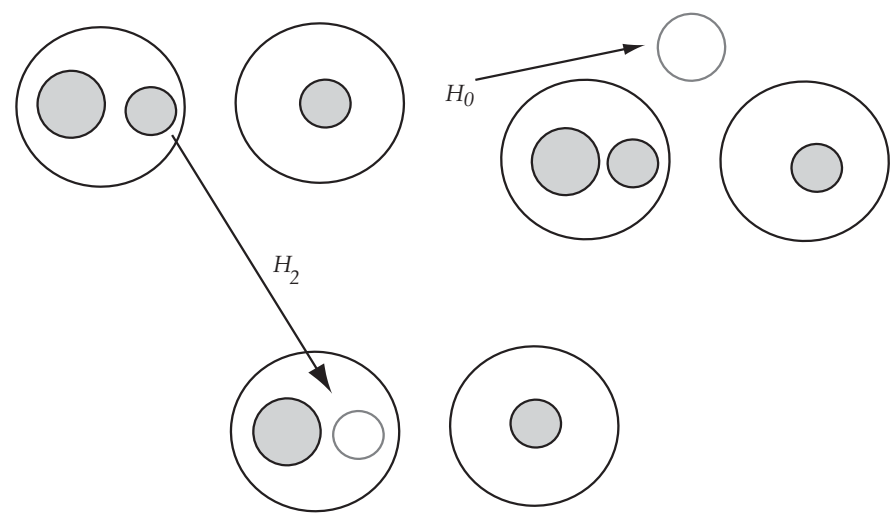

Figure 2. Attaching a 0-, 2-handle. The shaded areas indicate holes.

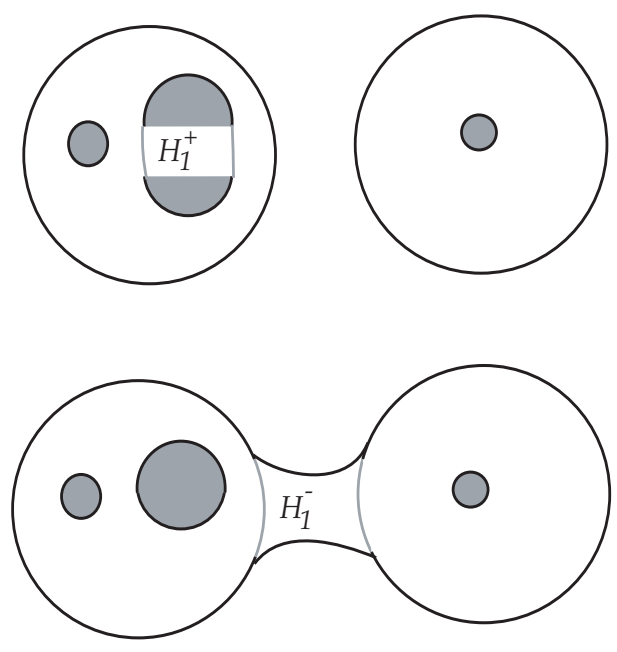

Figure 3. Admissible 1-handle attachments.

The level function associated to $f$ is the bijection $\ell_{f}$ from the set of critical points of $f$ to $[\nu(f)]$ which associates to each critical point $p$ the number of critical points $q$ such that $f(q) \leqslant f(p)$.

For every regular value $c$ of $f, \min f<c<\max f$, the components of the sublevel set $\{f \leqslant c\}$ are spheres with some disks removed. As we cross a critical value, the sublevel set $\{f \leqslant c\}$ is modified by a handle attachment.

A 0-handle attachment $H_{0}$ corresponds to crossing a local minimum of $f$. The sublevel set acquires a new component diffeomorphic to a closed disk (see Figure 2). A 2-handle attachment $\mathrm{H}_{2}$ corresponds to crossing a local maximum of $f$ and consists of attaching a 2-disk to a boundary component of $\{f \leqslant c\}$.

Crossing a saddle point has the effect of attaching a 1-handle. There are three different ways of attaching a 1-handle in an orientation-preserving fashion so that no Möbius band is created in the process, that is, we do not produce embedded circles with nontrivial normal bundle:

(i) $H_{1}^{+}$: the handle is attached to the same boundary component of the sublevel set (see Figure 3);

(ii) $H_{1}^{-}$: the handle is attached to different components of the sublevel set (see Figure 3); and

(iii) $H_{1}^{*}$ : the handle is attached to different boundary components of the same connected component of the sublevel set (see Figure 4). 


\section{I. NicOlAESCU}

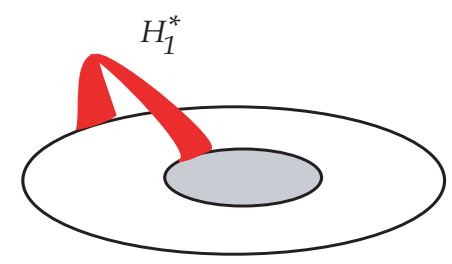

Figure 4. Inadmissible 1-handle attachment.

Lemma 3.1. For any excellent Morse function $f: S^{2} \rightarrow \mathbb{R}$ there are no $H_{1}^{*}$ handle attachments.

Proof. For any regular value of $c$ we set $X^{c}:=\{f \leqslant c\}$, and we denote by $\rho_{\mathrm{c}}$ the dimension of the cokernel of the natural morphism

$$
H_{1}\left(\partial X^{c}, \mathbb{R}\right) \rightarrow H_{1}\left(X^{c}, \mathbb{R}\right) .
$$

Consider a slicing $a_{0}<a_{1}<\cdots<a_{\nu}$ of $f$. The conclusion of the lemma follows from the following two elementary observations:

- $\rho_{a_{1}}=0=\rho_{a_{\nu-1}}$;

- $\rho_{a_{i+1}} \geqslant \rho_{a_{i}}$, with equality if and only if the passage from $X^{a_{i}}$ to $X^{a_{i+1}}$ is through a handle attachment of type $H_{0}, H_{1}^{ \pm}, H_{2}$ (an $H_{1}^{*}$ attachment produces a 1-cycle in the interior of the new sublevel set which is not homologous to any cycle on the boundary of that new sublevel set; see Figure 4).

To every regular value $\min f<c<\max f$ we associate a vector

$$
\mathbf{b}=\mathbf{b}(c):=\left(b_{0}\left(X^{c}\right), b_{1}\left(X^{c}\right)+1\right)
$$

where

$$
X^{c}=\{f \leqslant c\}, \quad b_{k}\left(X^{c}\right):=\operatorname{dim}_{\mathbb{R}} H_{k}\left(X^{c}, \mathbb{R}\right) .
$$

As the only admissible handle attachments are $H_{0}, H_{2}, H_{1}^{ \pm}$we conclude that, as we cross a critical value, the vector $\mathbf{b}$ undergoes one of the changes below

$$
\begin{array}{cc}
\mathbf{b} \stackrel{H_{0}}{\longrightarrow} \mathbf{b}+(1,0), & \mathbf{b} \stackrel{H_{2}}{\longrightarrow} \mathbf{b}+(0,-1), \\
\mathbf{b} \stackrel{H_{1}^{+}}{\longrightarrow} \mathbf{b}+(0,1), & \mathbf{b} \stackrel{H_{1}^{-}}{\longrightarrow} \mathbf{b}+(-1,0) .
\end{array}
$$

The combinatorics of an excellent Morse function on $S^{2}$ can be conveniently encoded using Morse trees. To describe these objects we need to introduce some combinatorial terminology.

In the sequel, a graph will mean a finite one-dimensional CW-complex, and a tree is a contractible graph. The 0-cells are called vertices, and the 1-cells edges. A labeling of a graph is an injection from the set of vertices to the set of real numbers. For every graph $\Gamma$ with vertex set $\mathcal{V}$, and every labeling $\varphi$ of $\Gamma$ we define the level function associated to $\varphi$

$$
\ell_{\varphi}: \mathcal{V} \rightarrow \mathbb{Z}, \quad \ell_{\varphi}(v):=\#\{u \in \mathcal{V}(\Gamma) ; \varphi(u) \leqslant \varphi(v)\} .
$$

A vertex $v$ is said to be higher than a vertex $u$ (with respect to $\varphi$ ) if $\varphi(v)>\varphi(u)$.

Definition 3.2. (a) A Morse tree order $n$ is a pair $(\Gamma, \varphi)$, where $\Gamma$ is a tree with vertex set $\mathcal{V}(\Gamma)$ of cardinality $2 n+2$, and $\varphi: \mathcal{V}(\Gamma) \rightarrow \mathbb{R}$ is a labeling such that the following hold.

(a1) Every vertex of $\Gamma$ has degree one or three. We will refer to the degree-three vertices as nodes.

(a2) Every node has at least one lower neighbor and at least one higher neighbor. 

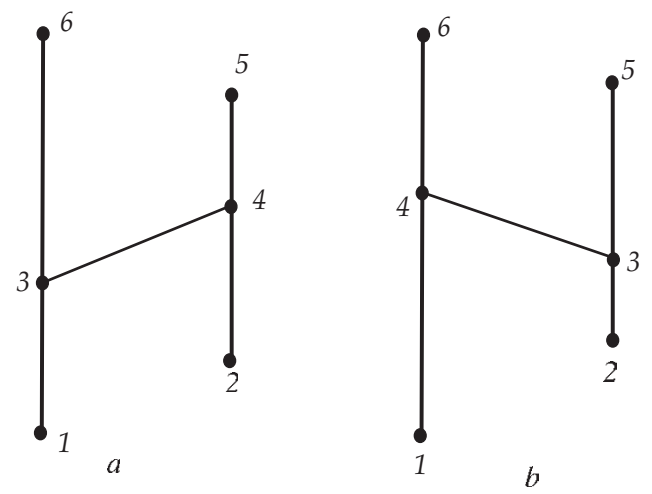

FiguRE 5. Morse trees of order two.

A degree-one vertex is called a maximum/minimum if it is higher/lower than its unique neighbor. We denote by $\mathcal{N}(\Gamma) \subset \mathcal{V}(\Gamma)$ the set nodes, and by $\mathcal{V}_{\max / \min }(\Gamma)$ the set of maxima/minima.

(b) Two Morse trees $\left(\Gamma_{i}, \varphi_{i}\right), i=0,1$ are said to be isomorphic, $\left(\Gamma_{0}, \varphi_{0}\right) \cong\left(\Gamma_{1}, \varphi_{1}\right)$, if there exists a bijection

$$
\beta: \mathcal{V}\left(\Gamma_{0}\right) \rightarrow \mathcal{V}\left(\Gamma_{1}\right)
$$

such that $u, v \in \mathcal{V}\left(\Gamma_{0}\right)$ are neighbors in $\Gamma_{0}$ if and only if $\beta(u), \beta(v)$ are neighbors in $\Gamma_{1}$ and

$$
\ell_{\varphi_{1}}(\beta(u))=\ell_{\varphi_{0}}(u), \quad \text { for all } u \in \Gamma_{0} .
$$

Note that the order of a Morse tree $\Gamma$ is equal to the number of nodes. We can embed a Morse tree $(\Gamma, \varphi)$ in $\mathbb{R}^{3}$ so that the following hold.

- The $z$ coordinates of the vertices are equal to the labels.

- The edges are smoothly embedded arcs, and the restriction of the function $z$ to each arc has no critical points.

We refer to a such a description of a Morse tree as an Euclidean embedding. In Figure 5 we depict Euclidean embeddings of several Morse trees of order two.

To any excellent Morse function $f: S^{2} \rightarrow \mathbb{R}$ we associate its Reeb graph $\mathcal{R}_{f}$ (see [BF04, Kud99, Kul98, Ree46, Sha03]) constructed as follows.

Define an equivalence relation $\sim_{f}$ on $S^{2}$ by declaring two points $p$ and $q$ to be equivalent if they lie in the same path component of a level set of $f$. As a topological space, $\mathcal{R}_{f}$ is the quotient $S^{2} / \sim_{f}$ equipped with the quotient topology. The space $\mathcal{R}_{f}$ is contractible, and it has a natural structure of one-dimensional $C W$-complex, with one vertex for every critical point of $f$. In other words, $\mathcal{R}_{f}$ is a tree.

The function $f$ defines a labeling of $\mathcal{R}_{f}$, and the pair $\left(\mathcal{R}_{f}, f\right)$ is a Morse tree called the Morse tree associated to $f$. The order of the Morse tree is equal to the order of the function $f$. We have the following result (see [Kul98, Theorem 2] and [Sha03, Theorem 6.2]).

Theorem 3.3. Two excellent Morse functions $f, g: S^{2} \rightarrow \mathbb{R}$ are geometrically equivalent if and only if the associated Morse trees $\left(\mathcal{R}_{f}, f\right)$ and $\left(\mathcal{R}_{g}, g\right)$ are isomorphic.

We can recover the geometric equivalence class of $f$ from its associated Morse tree $\left(\mathcal{R}_{f}, f\right)$ as follows. Consider an Euclidean embedding of the Morse tree. Then the boundary $M$ of a thin tubular neighborhood of the tree is diffeomorphic to $S^{2}$ and the restriction of the height function $z$ to $M$ is an excellent Morse function geometrically equivalent to $f$. The geometric equivalence class of 


\section{I. NicOlAESCU}
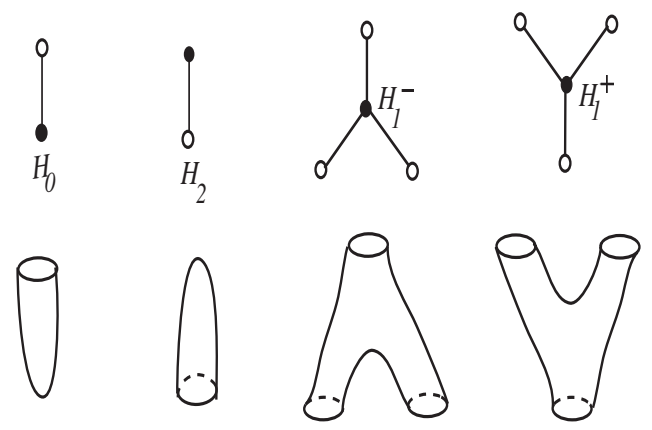

FiguRE 6 . The elementary cobordism corresponding determined by the critical values.

this function depends only on the isomorphism class of the Morse tree. We say that two Morse tree are homologically (respectively topologically) equivalent if their associated Morse functions are so. Observe that the two Morse trees in Figure 5 are topologically equivalent yet they are not isomorphic.

Remark 3.4. If $c$ is a critical value of an excellent Morse function $f: S^{2} \rightarrow \mathbb{R}$, then according to [Mil65, ch. 3] for $\varepsilon>0$ sufficiently small the region $\{|f-c| \leqslant \varepsilon\} \subset S^{2}$ is an elementary cobordism, that is, it is diffeomorphic to a disjoint union of cylinders $[c-\varepsilon, c+\varepsilon] \times S^{1}$ and one of the four surfaces with boundary depicted in Figure 6, where the upper boundary is contained in the level set $\{f=c+\varepsilon\}$ and the lower boundary is contained in the level set $\{f=c-\varepsilon\}$.

The vertices of a Morse tree are of the four types $H_{0}, H_{1}^{ \pm}, H_{2}$ and correspond to the four types of elementary cobordisms that can occur in a slicing of an excellent Morse function $f: S^{2} \rightarrow \mathbb{R}$ (see Figure 6).

The height function $z$ on each of the four surfaces embedded in $\mathbb{R}^{3}$ depicted in Figure 6 is an excellent Morse function which is constant on the upper and lower boundary. The heart of Theorem 3.3 is the following elementary fact: any Morse function on one of these four surfaces which has a unique critical point and it is constant on the upper and on the lower boundary is obtained from the height function via an orientation-preserving diffeomorphism of the surface which is identity on the boundary and an orientation-preserving diffeomorphism of $\mathbb{R}$.

\section{Counting homology equivalence classes}

We denote by $\mathcal{H}_{n}$ the set of homology equivalence classes of Morse functions $f: S^{2} \rightarrow \mathbb{R}$ of order $n$, and we set $h(n):=\# \mathcal{H}_{n}$. Suppose that $f: S^{2} \rightarrow \mathbb{R}$ is an excellent Morse function of order $n$, and $a_{0}<a_{1}<\cdots<a_{2 n+2}$ is a slicing of $f$.

To the function $f$ we now associate using (3.1) the sequence of lattice points

$$
P_{i}=\left(b_{0, i}, b_{1, i}+1\right) \in \mathbb{Z}_{>0}^{2}, \quad b_{k, i}:=\operatorname{dim} H_{k}\left(\left\{f \leqslant a_{i}\right\}, \mathbb{R}\right), \quad i=1, \ldots, 2 n+1 .
$$

Set

$$
E:=(1,0), \quad W:=-E, \quad N:=(0,1), \quad S:=-N, \quad \mathcal{D}:=\{E, W, N, S\} .
$$

Observe that $P_{1}=P_{2 n+1}=(1,1)$, and for every $i$ we have $P_{i+1}-P_{i} \in \mathcal{D}$. We regard the sequence $\mathcal{P}_{f}=\left\{P_{1}, P_{1}, \ldots, P_{2 n+1}\right\}$ as a lattice path of length $2 n$ in the interior of the first quadrant which starts and ends at $(1,1)$, and such that every step $P_{i} \rightarrow P_{i+1}$ has length one, and it is performed in one of the four possible lattice directions at $P_{i}$, East $(E)$, North $(N)$, West $(W)$, South $(S)$. 
We denote by $\mathcal{L}_{n}$ the set of such paths. Note that

$$
f \sim_{h} g \Longleftrightarrow \mathcal{P}_{f}=\mathcal{P}_{g},
$$

so that we have an injection

$$
\mathcal{H}_{n} \rightarrow \mathcal{L}_{n}, \quad f \mapsto \mathcal{P}_{f} .
$$

Lemma 4.1. The above map $\mathcal{H}_{n} \rightarrow \mathcal{L}_{n}$ is a bijection, so that $h(n)=\# \mathcal{L}_{n}$.

Proof. Given a lattice path $\mathcal{P} \in \mathcal{L}_{n}$ we need to produce a Morse function $f$ of order $n$ such that $\mathcal{P}_{f}=\mathcal{P}$. To construct such a function we use the following dictionary:

$$
E \longleftrightarrow H_{0}, \quad W \longleftrightarrow H_{1}^{-}, \quad N \longleftrightarrow H_{1}^{+}, \quad S \longleftrightarrow H_{2}
$$

We find it convenient to refer to the four types of handle attachments using the symbols $E, W, N, S$.

To every lattice point $P=(a, b) \in \mathbb{Z}_{>0}^{2}$ we can associate (non-uniquely) a surface $\Sigma_{P}$ which is a disjoint union of $a$ disks with a total number of $b-1$ holes in them. Note that we can perform a surgery of type $T \in \mathcal{D}$ on $\Sigma_{P}$ if and only if the neighbor of $P$ in the direction $T$ also lies in the interior of the first quadrant.

Thus, the path $\mathcal{P}$ describes a succession of handle attachments, starting with a disk and ending with a disk. As explained in [Mil65, Theorems 3.12 and 3.13], for any sequence of handle attachments there exists an excellent Morse function such that the successive topological changes in its sublevel sets are described by the sequence of handle attachments.

Theorem 4.2. The number of homological equivalence classes of excellent Morse functions $f: S^{2} \rightarrow$ $\mathbb{R}$ of order $n$ is

$$
h(n)=C_{n} \cdot C_{n+1} .
$$

Proof. We present a computation of $\# \mathcal{L}_{n}$ based on a reflection trick of Gessel-Zeilberger, which highlights the role of the Coxeter group $\mathbb{Z} / 2 \times \mathbb{Z} / 2$. For a different, more elementary proof of the equality $\# \mathcal{L}_{n}=C_{n} \cdot C_{n+1}$ we refer to [Guy01].

Define a lattice path to be a finite sequence of points $\gamma=\left\{P_{0}, \ldots, P_{L}\right\} \subset \mathbb{Z}^{2}$ such that $P_{i+1}-P_{i} \in \mathcal{D}$. The integer $L$ is called the length of the path and it is denoted by $L(\gamma)$. The path is called confined if all of the points $P_{i}$ are in the interior of the first quadrant, $\mathbb{Z}_{>0} \times \mathbb{Z}_{>0}$. A path is called bad if it is not confined.

For every integer $n$ we denote by $\mathcal{P}(P, Q ; n)$ the set of paths of length $n$ starting at $P$ and ending at $Q$. We let $\mathcal{P}_{\mathrm{c}}$ and $\mathcal{P}_{\mathrm{b}}$ denote the subsets consisting of confined and bad paths, respectively. We set

$$
\begin{gathered}
N(P, Q ; n):=\# \mathcal{P}(P, Q ; n), \quad N_{\mathrm{c}}(P, Q ; n):=\# \mathcal{P}_{\mathrm{c}}(P, Q ; n), \\
N_{\mathrm{b}}(P, Q ; n)=\# \mathcal{P}_{\mathrm{b}}(P, Q ; n) .
\end{gathered}
$$

The above discussion shows that

$$
h(n)=N_{\mathrm{c}}\left(P_{1}, P_{1} ; 2 n\right), \quad P_{1}=(1,1) .
$$

Consider two points $P, Q$ in the first quadrant. Denote by $r_{1}, r_{2}: \mathbb{R}^{2} \rightarrow \mathbb{R}^{2}$ the reflections

$$
r_{1}\left(t_{1}, t_{2}\right)=\left(-t_{1}, t_{2}\right), \quad r_{2}\left(t_{1}, x_{2}\right)=\left(t_{1},-t_{2}\right) .
$$

These reflections generate the Klein group

$$
K=\left\{\mathbb{1}, r_{1}, r_{2}, r=r_{1} r_{2}\right\} \cong \mathbb{Z} / 2 \times \mathbb{Z} / 2 .
$$

Then, arguing as in the proof of [GZ92, Theorem 1] we deduce that

$$
N_{\mathrm{b}}(P, Q ; n)+N_{\mathrm{b}}(P, r(Q) ; n)=N_{\mathrm{b}}\left(P, r_{1}(Q) ; n\right)+N_{\mathrm{b}}\left(P, r_{2}(Q) ; n\right) .
$$




\section{I. NiCOLAESCU}

Since $r(Q), r_{1}(Q), r_{2}(Q)$, are not in the first quadrant we deduce that

$$
N_{\mathrm{b}}(P, \rho(Q))=N(P, \rho(Q)), \quad \text { for all } \rho \in\left\{r, r_{1}, r_{2}\right\},
$$

and thus

$$
N_{\mathrm{c}}(P, Q ; n)=N(P, Q ; n)+N(P, r(Q) ; n)-N\left(P, r_{1}(Q) ; n\right)-N\left(P, r_{2}(Q) ; n\right) .
$$

Observing that $N(P, Q ; n)=N(\mathbf{0}, Q-P ; n)$ we deduce that

$$
N_{\mathrm{c}}\left(P_{1}, P_{1} ; n\right)=N(\mathbf{0}, \mathbf{0} ; n)+N\left(\mathbf{0},-2\left(e_{1}+e_{2}\right) ; n\right)-N\left(\mathbf{0},-2 e_{2} ; n\right)-N\left(\mathbf{0},-2 e_{1} ; n\right) .
$$

For $t=\left(t_{1}, t_{2}\right)$, and $\mathbf{x}=\left(x_{1}, x_{2}\right) \in \mathbb{Z}^{2}$, we set $t^{\mathbf{x}}=t_{1}^{x_{1}} t_{2}^{x_{2}}$. Consider the step polynomial

$$
\mathcal{S}(t):=\sum_{\mathbf{x} \in \mathcal{D}} t^{\mathbf{x}}=t_{1}+t_{2}+t_{1}^{-1}+t_{2}^{-1} \in \mathbb{Z}\left[\left[t_{1}, t_{2}, t_{1}^{-1}, t_{2}^{-1}\right]\right] .
$$

For every $\mathrm{x} \in \mathbb{Z}^{2}$ define

$$
\mathcal{C}_{\mathbf{x}}: \mathbb{C}\left[\left[t_{1}, t_{2}, t_{1}^{-1}, t_{2}^{-1}\right]\right] \rightarrow \mathbb{C}, \quad A=\sum_{\mathbf{y} \in \mathbb{Z}^{2}} A_{\mathbf{y}} t^{\mathbf{y}} \longmapsto \mathcal{C}_{\mathbf{x}}(A)=A_{\mathbf{x}} .
$$

In other words, $\mathcal{C}_{\mathbf{x}}$ associates to each Laurent polynomial the coefficient of $t^{\mathrm{x}}$ in that polynomial. Observe that for every $\mathbf{x} \in \mathbb{Z}^{2}$ we then have

$$
N(\mathbf{0}, \mathbf{x} ; n)=\mathcal{C}_{\mathbf{x}}\left(\mathcal{S}(t)^{n}\right),
$$

If we regard $t_{j}$ as a complex parameter, $t_{j}=\left|t_{j}\right| e^{i \theta_{j}}$, we have the integral formula

$$
\mathrm{C}_{\mathbf{x}}(A)=\oint A(t) t^{-\mathbf{x}}:=\frac{1}{4 \pi^{2}} \int_{\left|t_{1}\right|=\left|t_{2}\right|=1} A(t) t^{-\mathbf{x}} d \theta_{1} d \theta_{2}
$$

Using (4.1) we conclude that

$$
N_{\mathrm{c}}\left(P_{1}, P_{1} ; n\right)=\oint \mathcal{S}(t)^{n}\left(1-t_{1}^{2}-t_{2}^{2}+t_{1}^{2} t_{2}^{2}\right)=\oint \mathcal{S}(t)^{n}\left(t_{1}^{2}-1\right)\left(t_{2}^{2}-1\right) .
$$

Hence,

$$
\sum_{n \geqslant 0} h(n) z^{2 n}=\oint \frac{\left(t_{1}^{2}-1\right)\left(t_{2}^{2}-1\right)}{1-z \mathcal{S}}=\oint \underbrace{\frac{\left(t_{1}^{2}-1\right)\left(t_{2}^{2}-1\right)}{1-z\left(t_{1}+t_{1}^{-1}+t_{2}+t_{2}^{-1}\right)}}_{=: K(z, t)}
$$

Observe that

$$
K(z, t)=\frac{t_{1} t_{2}\left(t_{1}^{2}-1\right)\left(t_{2}^{2}-1\right)}{t_{1} t_{2}-z\left(t_{1} t_{2}+1\right)\left(t_{1}+t_{2}\right)} \quad \text { and } \quad K\left(z, t_{1}, t_{2}\right)=K\left(z, t_{2}, t_{1}\right) .
$$

We write

$$
u:=t_{1}+t_{2}, \quad v:=t_{1} t_{2},
$$

and we deduce that

$$
\begin{aligned}
K(z, t) & =\frac{v\left(v^{2}-u^{2}+2 v+1\right)}{v-z u(v+1)}=\left(v^{2}-u^{2}+2 v+1\right) \cdot \frac{1}{1-z u(1+1 / v)} \\
& =\left(v^{2}-u^{2}+2 v+1\right) \cdot \sum_{n \geqslant 0} z^{n} u^{n}\left(1+v^{-1}\right)^{n} \\
& =\sum_{n \geqslant 0} z^{n} \sum_{k=0}^{n}\left(\begin{array}{l}
n \\
k
\end{array}\right)\left(v^{2}-u^{2}+2 v+1\right) u^{n} v^{-k} \\
& =\sum_{n \geqslant 0} z^{n} \sum_{k=0}^{n}\left(\begin{array}{l}
n \\
k
\end{array}\right)\left(u^{n} v^{2-k}-u^{n+2} v^{-k}+2 u^{n} v^{1-k}+u^{n} v^{-k}\right) .
\end{aligned}
$$


Fortunately, very few terms in this sum contribute to (4.2) since

$$
\oint u^{n} v^{-k}=0, \quad \text { for all } n \neq 2 k \text {. }
$$

Moreover

We deduce that

$$
\oint u^{2 m} v^{-m}=\left(\begin{array}{c}
2 m \\
m
\end{array}\right)
$$

$$
\oint K(z, t) d t=\sum_{m \geqslant 0} z^{2 m} \sum_{k=0}^{2 m}\left(\begin{array}{c}
2 m \\
k
\end{array}\right) \oint\left(u^{2 m} v^{2-k}-u^{2 m+2} v^{-k}+2 u^{2 m} v^{1-k}+u^{2 m} v^{-k}\right) .
$$

This shows that for every $m \geqslant 0$ we have

$$
h(m)=\underbrace{\left(\begin{array}{c}
2 m \\
m+2
\end{array}\right)\left(\begin{array}{c}
2 m \\
m
\end{array}\right)}_{a}-\underbrace{\left(\begin{array}{c}
2 m+2 \\
m+1
\end{array}\right)\left(\begin{array}{c}
2 m \\
m+1
\end{array}\right)}_{b}+2 \underbrace{\left(\begin{array}{c}
2 m \\
m
\end{array}\right)\left(\begin{array}{c}
2 m \\
m+1
\end{array}\right)}_{c}+\underbrace{\left(\begin{array}{c}
2 m \\
m
\end{array}\right)\left(\begin{array}{c}
2 m \\
m
\end{array}\right)}_{d} .
$$

Now observe that

$$
\begin{aligned}
& a+c=\left(\begin{array}{c}
2 m \\
m+2
\end{array}\right)\left(\begin{array}{c}
2 m \\
m
\end{array}\right)+\left(\begin{array}{c}
2 m \\
m
\end{array}\right)\left(\begin{array}{c}
2 m \\
m+1
\end{array}\right)=\left(\begin{array}{c}
2 m \\
m
\end{array}\right)\left(\begin{array}{c}
2 m+1 \\
m+2
\end{array}\right), \\
& c+d=\left(\begin{array}{c}
2 m \\
m
\end{array}\right)\left(\begin{array}{c}
2 m \\
m+1
\end{array}\right)+\left(\begin{array}{c}
2 m \\
m
\end{array}\right)\left(\begin{array}{c}
2 m \\
m
\end{array}\right)=\left(\begin{array}{c}
2 m \\
m
\end{array}\right)\left(\begin{array}{c}
2 m+1 \\
m+1
\end{array}\right) .
\end{aligned}
$$

The sum of the right-hand-sides of the above equalities is

$$
a+2 c+d=\left(\begin{array}{c}
2 m \\
m
\end{array}\right)\left(\begin{array}{c}
2 m+1 \\
m+2
\end{array}\right)+\left(\begin{array}{c}
2 m \\
m
\end{array}\right)\left(\begin{array}{c}
2 m+1 \\
m+1
\end{array}\right)=\left(\begin{array}{c}
2 m \\
m
\end{array}\right)\left(\begin{array}{c}
2 m+2 \\
m+2
\end{array}\right) .
$$

Hence,

$$
\begin{aligned}
h(m)=a+2 c+d-b & =\left(\begin{array}{c}
2 m \\
m
\end{array}\right)\left(\begin{array}{c}
2 m+2 \\
m+2
\end{array}\right)-\left(\begin{array}{c}
2 m+2 \\
m+1
\end{array}\right)\left(\begin{array}{c}
2 m \\
m+1
\end{array}\right) \\
& =\frac{1}{(m+2)(m+1)}\left(\begin{array}{c}
2 m+2 \\
m+1
\end{array}\right)\left(\begin{array}{c}
2 m \\
m
\end{array}\right)=C_{m+1} C_{m} .
\end{aligned}
$$

Remark 4.3. The sequence $C_{n} C_{n+1}$ is the $A 005568$ sequence in Sloane's on-line encyclopedia, and has many other interesting combinatorial interpretations.

\section{A recursive construction of geometric equivalence classes}

In this section we produce a two-parameter recursion formula for the number of (isomorphism classes of) Morse trees. One of the parameters will be the order of the tree and the other parameter will be the level of the lowest node.

For every subset $C \subset \mathbb{R}$ we denote by $\mathcal{F}_{C}$ the set of Morse trees $(\Gamma, \varphi)$ such that $\varphi(\mathcal{V}(\Gamma))=C$. We say that $C$ is the discriminant set of $\varphi$. We define $\mathcal{F}_{C}(m) \subset \mathcal{F}_{C}$ to be the subset consisting of Morse trees such that the lowest $m$ vertices are local minima. Let

$$
\mathcal{F}_{C}^{*}(m):=\mathcal{F}_{C}(m) \backslash \mathcal{F}_{C}(m+1) .
$$

Observe that $\# \mathcal{F}_{C}(m)=\# \mathcal{F}_{C^{\prime}}(m)$ if $\# C=\# C^{\prime}$. We set

$$
\mathcal{F}_{n}(m):=\mathcal{F}_{[2 n+2]}(m), \quad F_{n}(m):=\# \mathcal{F}_{n}(m), \quad f_{n}(m)=F_{n}(m)-F_{n}(m+1) .
$$

Note that $f_{n}(m)$ is equal to the number of Morse trees of order $n$ such that the lowest $m$ vertices are local minima, while the $(m+1)$ vertex is a node. Since level of the lowest node of a Morse tree 


\section{I. NicOlAESCU}

of order $n$ is at most $n+1$, we deduce that

$$
F_{n}(m)=0, \quad \text { for all } m>n+1 \Longrightarrow f_{n}(n+1)=F_{n}(n+1) .
$$

Here $F_{n}(1)$ is precisely the number of Morse trees of order $n$ and

$$
F_{n}(m)=\sum_{k \geqslant m} f_{n}(k), \quad f_{n}(m)=0, \quad \text { for all } m>n+1 .
$$

Theorem 5.1. We have

$$
\begin{aligned}
f_{n}(m)= & \left(\begin{array}{c}
m \\
2
\end{array}\right) F_{n-1}(m-1) \\
& +\sum_{m_{0}+m_{1}=m-1} \sum_{n_{0}+n_{1}=n-1} \frac{m}{2}\left(\begin{array}{c}
m-1 \\
m_{1}
\end{array}\right)\left(\begin{array}{c}
2 n-m+1 \\
2 n_{0}-m_{0}+1
\end{array}\right) F_{n_{0}}\left(m_{0}+1\right) F_{n_{1}}\left(m_{1}+1\right) .
\end{aligned}
$$

Proof. If $(\Gamma, \varphi) \in \mathcal{F}_{n}^{*}(m)$, then $\varphi=\ell_{\varphi}$, and the level $m+1$ vertex of $\Gamma$ is a node. It can only be one of two types:

- negative type, if $m+1$ has two lower neighbors;

- positive type, if $m+1$ has a unique lower neighbor and thus two higher neighbors.

Correspondingly, we obtain a partition $\mathcal{F}_{n}^{*}(m)=\mathcal{F}_{n}^{+}(m) \sqcup \mathcal{F}_{n}^{-}(m)$. We set $F_{n}^{ \pm}(m):=\# \mathcal{F}_{n}^{ \pm}(m)$ so that

$$
f_{n}(m)=\# \mathcal{F}_{n}^{*}(m)=F_{n}^{+}(m)+F_{n}^{-}(m)
$$

We discuss two cases.

Case $\mathbf{C}_{+}$. Suppose that $(\Gamma, \varphi) \in \mathcal{F}_{n}^{+}(m)$.

Denote by $v_{0}, v_{1}$ the two higher neighbors of the $(m+1)$ th vertex, and by $u$ its unique lower neighbor. For $\epsilon=0,1$ we set

$$
k_{\epsilon}:=\varphi\left(v_{\epsilon}\right), \quad k:=\varphi(u), \quad k_{0}, k_{1}>m+1>k .
$$

Denote by $\Gamma^{u}$ the graph obtained from $\Gamma$ by removing the vertices $u, m+1$, and the edges at these points. Denote by $\Gamma_{\epsilon}^{u}$ the component of $\Gamma^{u}$ containing the vertex $v_{\epsilon}$, and define $\bar{\Gamma}_{\epsilon}$ by setting

$$
\mathcal{V}\left(\bar{\Gamma}_{\epsilon}\right):=\mathcal{V}\left(\Gamma_{\epsilon}^{u}\right) \sqcup\left\{r_{\epsilon}\right\}
$$

where the new vertex $r_{\epsilon}$ has only one neighbor in $\bar{\Gamma}_{\epsilon}$, the vertex $v_{\epsilon}$; see Figure 7 .

Now define the labelings

$$
\varphi_{\epsilon}: \mathcal{V}\left(\bar{\Gamma}_{\epsilon}\right) \rightarrow \mathbb{R}, \quad \varphi_{\epsilon}\left(r_{\epsilon}\right)=m+1, \quad \varphi_{\epsilon}(w)=\varphi(w), \quad \text { for all } w \in \mathcal{V}\left(\Gamma_{\epsilon}^{u}\right)
$$

Denote by $M_{\epsilon}$ the subset of $[m]$ consisting of vertices which belong to the component $\Gamma_{\epsilon}^{u}$, and set $m_{\epsilon}:=\# M_{\epsilon}$. We have

$$
M_{0} \cup M_{1}=M_{k}=[m] \backslash\{\varphi(u)\}=[m] \backslash\{k\} \Longrightarrow m_{0}+m_{1}=m-1 .
$$

Let us define a 2 -coloring of a set $S$ to be a surjection $u: S \rightarrow\{0,1\}$. A 2-coloring is uniquely determined by the ordered pair $\left(S_{0}, S_{1}\right)=\left(u^{-1}(0), u^{-1}(1)\right.$. If we set

$$
A_{\epsilon}:=\mathcal{V}\left(\Gamma_{\epsilon}^{u}\right) \backslash M_{\epsilon}
$$

we deduce that $\left(A_{0}, A_{1}\right)$ is a 2 -coloring of $\{m+2, \ldots, 2 n+2\}$.

We see that $\left(\bar{\Gamma}_{\epsilon}, \varphi_{\epsilon}\right)$ is a Morse tree such that its first $m_{\epsilon}+1$ vertices are local minima. The discriminant set of $\varphi_{\epsilon}$ is

$$
C_{\epsilon}=\mathcal{V}\left(\Gamma_{\epsilon}^{u}\right) \cup\{m+1\}=A_{\epsilon} \sqcup M_{\epsilon} \sqcup\{m+1\},
$$



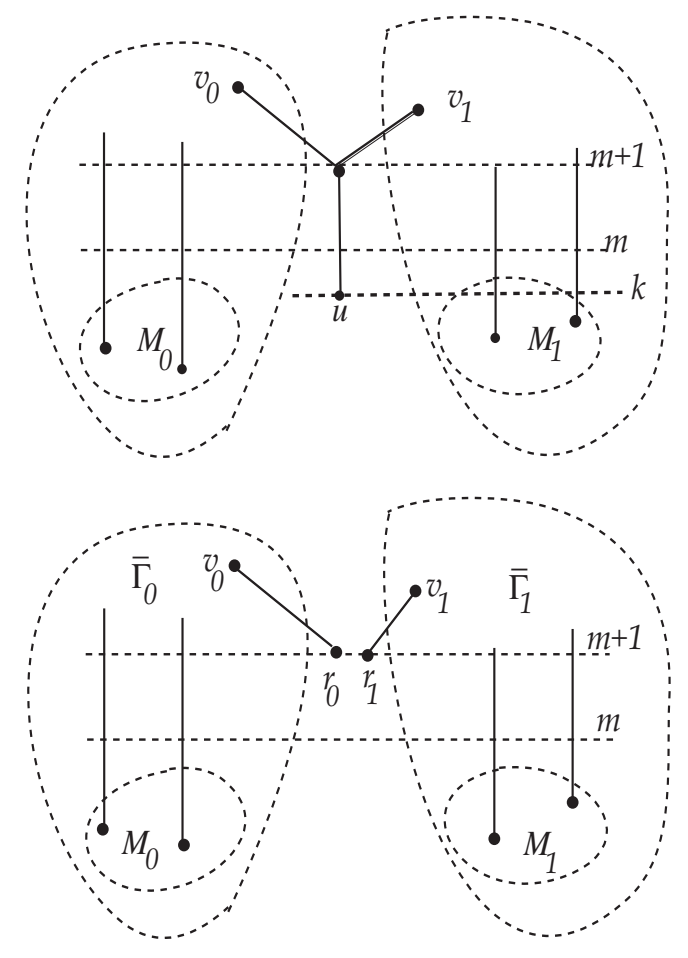

Figure 7 . Cutting a Morse tree along the lowest $H_{1}^{+}$-handle.

so that

$$
\bar{\Gamma}_{\epsilon} \in \mathcal{F}_{C_{\epsilon}}\left(m_{\epsilon}+1\right) .
$$

We conclude that the initial Morse tree $(\Gamma, \varphi)$ is uniquely determined by the following data.

- The integer $k=\varphi(u) \in\{1, \ldots, m\}$.

- The 2-coloring of $M_{k}=[m] \backslash\{k\}, M_{k}=M_{0} \sqcup M_{1}$, \# $M_{\epsilon}=m_{\epsilon}$.

- The 2-coloring $\left(A_{0}, A_{1}\right)$ of $\{m+2, \ldots, 2 n+2\}, a_{\epsilon}=\# A_{\epsilon}$.

- The choices of Morse trees $\left(\bar{\Gamma}_{\epsilon}, \varphi_{\epsilon}\right)$ with discriminant sets $C_{\epsilon}=M_{\epsilon} \sqcup A_{\epsilon} \sqcup\{m+1\}$, such that $\left(\bar{\Gamma}_{\epsilon}, \varphi_{\epsilon}\right) \in \mathcal{F}_{C_{\epsilon}}\left(m_{\epsilon}+1\right)$.

The tree $\bar{\Gamma}_{\epsilon}$ has $a_{\epsilon}+m_{\epsilon}+1$ vertices, and its order is $n_{\epsilon}=\frac{1}{2}\left(a_{\epsilon}+m_{\epsilon}-1\right)$. We deduce that $a_{\epsilon}+m_{\epsilon} \equiv 1 \bmod 2$, and

$$
a_{\epsilon}=2 n_{\epsilon}-m_{\epsilon}+1, \quad n_{0}+n_{1}=n-1 .
$$

We have thus produced a surjection

$$
\Psi: \bigsqcup_{k=1}^{m} \bigsqcup_{\left(C_{0}^{k}, C_{1}^{k}\right)} \mathcal{F}_{C_{0}^{k}}\left(m_{0}+1\right) \times \mathcal{F}_{C_{1}^{k}}\left(m_{1}+1\right) \longrightarrow \mathcal{F}_{n}(m)
$$

where the disjoint union is taken over all ordered pairs $\left(C_{0}^{k}, C_{1}^{k}\right)$ satisfying

$$
\begin{gathered}
C_{\epsilon}^{k}=M_{\epsilon}^{k} \sqcup A_{\epsilon} \sqcup\{m+1\}, \quad M_{0}^{k} \sqcup M_{1}^{k}=[m] \backslash\{k\} \\
A_{0} \sqcup A_{1}=\{m+2, \ldots, 2 n+2\}, \quad \# A_{\epsilon} \equiv \# M_{\epsilon}^{k}+1 \bmod 2 .
\end{gathered}
$$

We have a fixed-point-free involution on the left-hand-side of (5.3) defined by the bijections induced by the transposition of subscripts, $0 \longleftrightarrow 1$,

$$
\mathcal{F}_{C_{0}^{k}}\left(m_{0}+1\right) \times \mathcal{F}_{C_{1}^{k}}\left(m_{1}+1\right) \longrightarrow \mathcal{F}_{C_{1}^{k}}\left(m_{1}+1\right) \times \mathcal{F}_{C_{0}^{k}}\left(m_{0}+1\right) .
$$




\section{I. NiCOLAESCU}

The fibers of $\Psi$ are precisely the orbits of this involution. We deduce that

$$
F_{n}^{+}(m)=\frac{1}{2} \sum_{k} \sum_{\left(C_{0}^{k}, C_{1}^{k}\right)} \# \mathcal{F}_{C_{0}^{k}}\left(m_{0}+1\right) \times \# \mathcal{F}_{C_{1}^{k}}\left(m_{1}+1\right) .
$$

The integer $1 \leqslant k \leqslant m$ can be chosen in $m$ different ways. The 2 -coloring $\left(M_{0}, M_{1}\right)$ of $\{1, \ldots, m\} \backslash k$ can be chosen in $\left(\begin{array}{c}m-1 \\ m_{0}, m_{1}\end{array}\right)=\left(\begin{array}{c}m-1 \\ m_{0}\end{array}\right)$ ways. For every pair $\left(n_{0}, n_{1}\right)$ of nonnegative integers such that $n-1=n_{0}+n_{1}$, we can choose the 2 -coloring $\left(A_{0}, A_{1}\right)$ of $\{m+2, \ldots, 2 n+2\}$ in $\left(\begin{array}{c}2 n-m+1 \\ a_{0}, a_{1}\end{array}\right)=\left(\begin{array}{c}2 n-m+1 \\ a_{0}\end{array}\right)$ ways. Finally, the Morse tree $\bar{\Gamma}_{\epsilon} \in \mathcal{F}_{C_{\epsilon}}\left(m_{\epsilon}+1\right)$ can be chosen in $F_{n_{\epsilon}}\left(m_{\epsilon}+1\right)$ ways. We deduce that

$$
F_{n}^{+}(m)=\frac{m}{2} \sum_{m_{0}=0}^{m-1} \sum_{n_{0}=0}^{n-1}\left(\begin{array}{c}
m-1 \\
m_{0}
\end{array}\right)\left(\begin{array}{c}
2 n-m+1 \\
2 n_{0}-m_{0}+1
\end{array}\right) F_{n_{0}}\left(m_{0}+1\right) F_{n_{1}}\left(m_{1}+1\right) .
$$

Case $\mathbf{C}_{-}$. Suppose that $(\Gamma, \varphi) \in \mathcal{F}_{n}^{-}(m)$. In this case, the vertex $m+1$ has two lower neighbors $1 \leqslant k_{1}<k_{2} \leqslant m$, and a higher neighbor $K>m+1$. We can now produce a Morse tree $\left(\Gamma^{\prime}, \varphi^{\prime}\right)$ of order $n-1$ by removing the vertices $k_{2}, m+1$ and their incident edges, and then connecting $k_{1}$ and $K$ by an edge (see Figure 8 ). Thus,

$$
\mathcal{V}\left(\Gamma^{\prime}\right)=\mathcal{V}(\Gamma) \backslash\left\{k_{2}, m+1\right\} \subset \mathcal{V}(\Gamma) .
$$

The labeling $\varphi^{\prime}$ is defined by $\varphi^{\prime}:=\varphi \mid \mathcal{V}\left(\Gamma^{\prime}\right)$. The discriminant set of $\varphi^{\prime}$ is $[2 n+2] \backslash\left\{m+1, k_{2}\right\}$, and the lowest $m-1$ vertices are local minima. The Morse tree $(\Gamma, \varphi)$ is uniquely determined by the following data.

- The pair of integers $k_{1}<k_{2} \in\{1,2, \ldots, m\}$.

- The Morse tree $\left(\Gamma^{\prime}, \varphi^{\prime}\right)$ of order $n-1$, with discriminant set $[2 n+2] \backslash\left\{m+1, k_{2}\right\}$ whose lowest $m-1$ vertices are local minima.

We deduce

$$
F_{n}^{-}(m)=\left(\begin{array}{c}
m \\
2
\end{array}\right) F_{n-1}(m-1) .
$$

The equality (5.1) now follows from (5.2), (5.4) and (5.5).

Corollary 5.2. If $n \geqslant 1$, then

$$
F_{n}(n+1)=f_{n}(n+1)=\left(\begin{array}{c}
n+1 \\
2
\end{array}\right) F_{n-1}(n)
$$

Proof. Indeed, the second term in (5.1) is nontrivial if and only if $m_{0}+1 \leqslant n_{0}+1$ and $m_{1}+1 \leqslant n_{1}+1$. In particular, it vanishes when $m_{0}+m_{1}>n_{0}+n_{1}$, which is the case.

Remark 5.3. The set of isomorphism classes of Morse trees is equipped with a natural involution

$$
(\Gamma, \varphi) \longleftrightarrow(\Gamma,-\varphi)
$$

which we call Poincaré duality because it mimics the Poincaré duality. We say that a Morse tree is selfdual if it is isomorphic to its Poincaré dual.

We find the problem of computing the number of isomorphism classes of selfdual Morse trees, or even their asymptotics, very compelling. An understanding of the number of the fixed points of the above involution would lead to an understanding of the number of stable orbits of the action of the group $\operatorname{Diff}_{+}\left(S^{2}\right) \times \operatorname{Diff}(\mathbb{R})$ on the space $\mathcal{F}$ of smooth functions $S^{2} \rightarrow \mathbb{R}$.

Unfortunately, this problem has resisted all of our efforts. The number of such trees of very low order is recorded in the next example. 
Morse FUnCTIONS ON THE 2-SPHERE

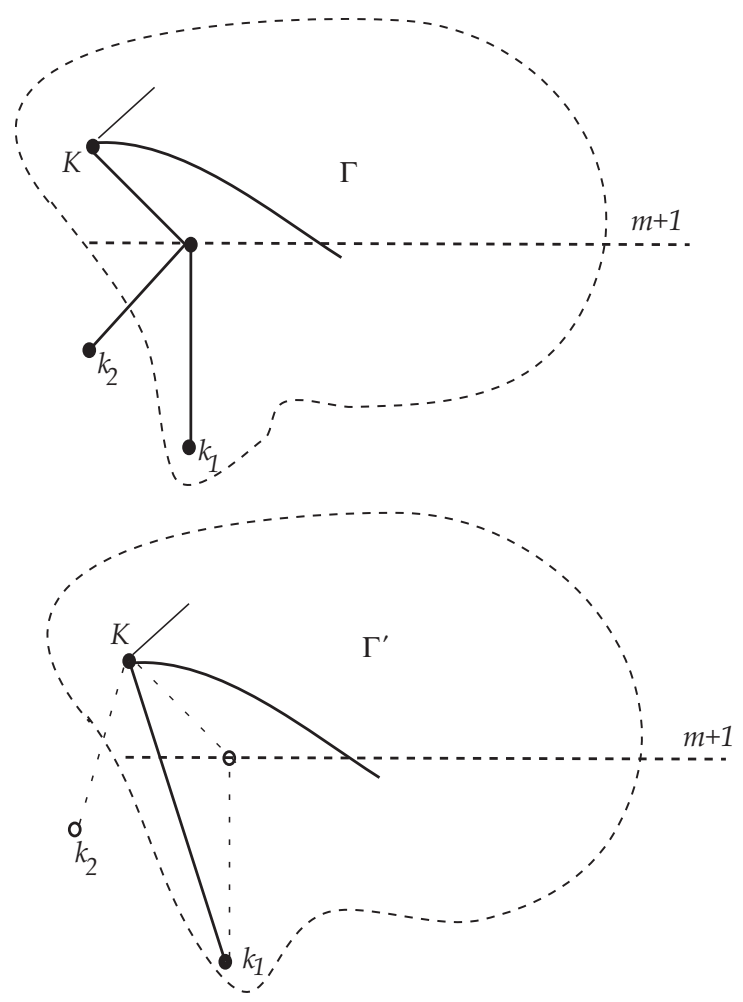

Figure 8. Cutting a Morse tree along the lowest $H_{1}^{-}$-handle.

$$
\left.\right|_{1} ^{2}
$$
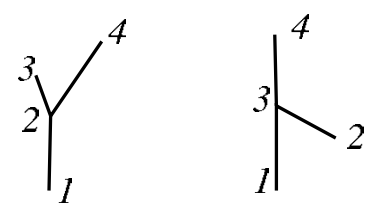

FiguRE 9. Morse trees with 2 and 4 vertices.

Example 5.4. Let $n=0$. In this case there are no nodes and we have

$$
F_{0}(1)=1, \quad F_{0}(m)=0, \quad \text { for all } m>1 .
$$

In Figure 9 we depict the only Morse tree of order zero.

Let $n=1$. Then Theorem 5.1 predicts

$$
f_{1}(1)=1, \quad f_{1}(2)=F_{0}(1)=1 \Longrightarrow F_{1}(1)=2, \quad F_{1}(2)=1 .
$$

The two Morse trees are depicted in Figure 9.

For $n=2, m=1$ we have

$$
f_{2}(1)=\frac{1}{2} \sum_{j=0}^{1}\left(\begin{array}{c}
4 \\
2 j+1
\end{array}\right) F_{j}(1) F_{1-j}(1)=4 F_{0}(1) F_{1}(1)=8 .
$$

Thus we have eight Morse trees with six vertices such that the second vertex is a node. They are depicted in Figure 10, where $(S):=$ selfdual. 


\section{I. NicOlAESCU}
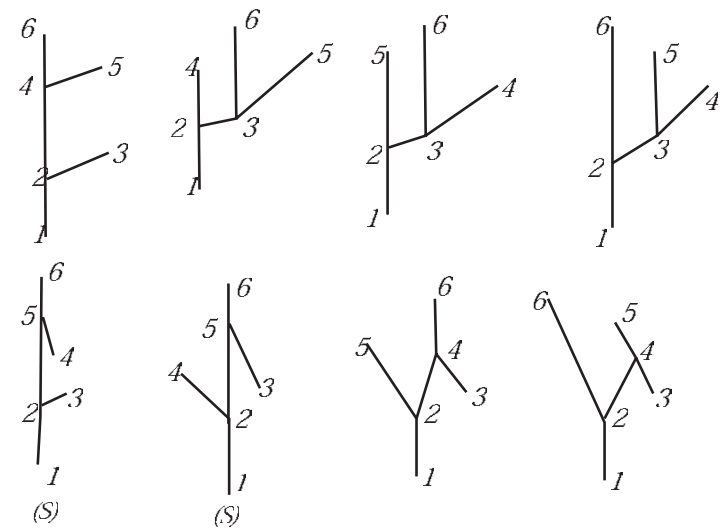

FiguRE 10. Morse trees with six critical points, and the second is a node.

For $n=2, m=2$ we have

$$
\begin{aligned}
f_{2}(2) & =F_{1}(1)+\sum_{j=0}^{1}\left(\begin{array}{c}
3 \\
j+1
\end{array}\right) F_{j}(j+1) f_{1-j}(2-j) \\
& =F_{1}(1)+\left(\begin{array}{l}
3 \\
2
\end{array}\right) F_{1}(2) F_{0}(1)+\left(\begin{array}{l}
3 \\
1
\end{array}\right) F_{0}(1) F_{1}(2)=2+3+3=8 .
\end{aligned}
$$

These trees are depicted in Figure 11, where $(S):=$ selfdual.

Finally, for $n=2$ and $m=3$ we have $f_{2}(3)=\left(\begin{array}{l}3 \\ 2\end{array}\right) F_{1}(2)=3$. These three Morse trees are depicted in Figure 12. Hence,

$$
F_{2}(1)=f_{2}(1)+f_{2}(2)+f_{2}(3)=8+8+3=19, \quad F_{2}(2)=11, \quad F_{2}(3)=3 .
$$

We list below the numbers of homology equivalence classes and geometric equivalence classes of Morse functions with $2 n+2$ critical points, $n \leqslant 9$. The number of geometric equivalence classes were computed using a simple Maple procedure based on the above recurrence.

Remark 5.5. (a) The numbers of geometric equivalence classes of Morse functions with at most 10 critical points were also determined by Arnold in [Arn06] and agree with the findings listed in Table 1.

(b) In [Nic06] we show that Theorem 5.1 leads to a positive answer to a question of Arnold. If $g(n)$ is the number of geometric equivalence classes of Morse functions on $S^{2}$ with $2 n+2$ critical points, then

$$
\lim _{n \rightarrow \infty} \frac{\log g(n)}{n \log n}=2
$$

The computations in the above example give an idea of the complexity of the above recurrence and suggest that it can be better organized. We do this in the next section.

\section{Generating functions}

We introduce new functions

$$
g(m, n):=f_{n}(m+1), \quad G(m, n):=F_{n}(m+1), \quad 0 \leqslant m \leqslant n,
$$

so that $g(m, n)=G(m, n)-G(m+1, n)$. 

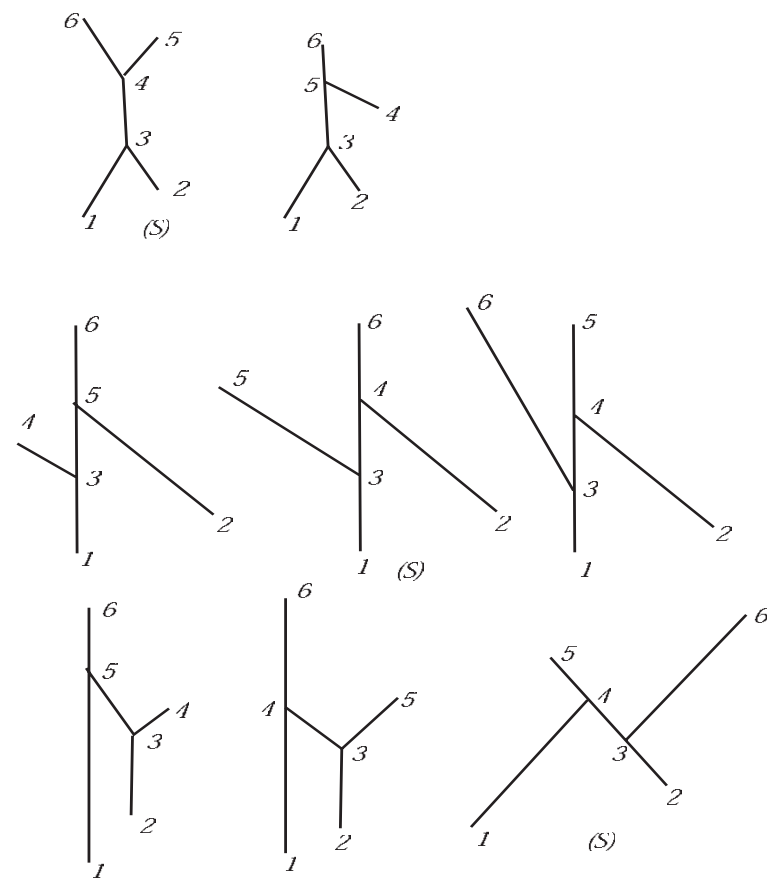

FiguRE 11. Morse trees with six vertices, and the first two are minima.
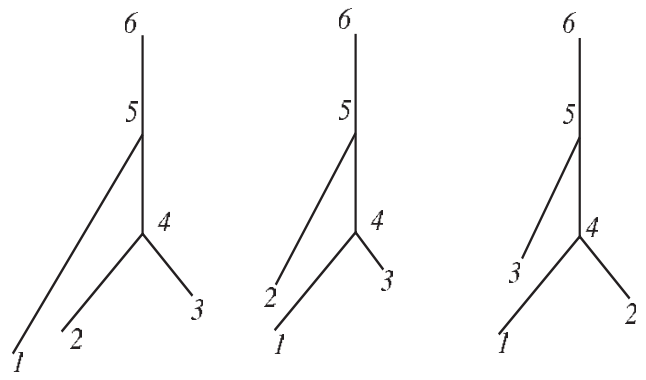

Figure 12. Morse trees with six vertices, three minima.

TABLE 1.

\begin{tabular}{rrrr}
\hline$n$ & $2 n+2$ & Homology classes & Geometric classes \\
\hline 0 & 2 & 1 & 1 \\
1 & 4 & 2 & 2 \\
2 & 6 & 10 & 19 \\
3 & 8 & 70 & 428 \\
4 & 10 & 588 & 17746 \\
5 & 12 & 5544 & 1178792 \\
6 & 14 & 56628 & 114892114 \\
7 & 16 & 613470 & 15465685088 \\
8 & 18 & 6952660 & 2750970320776 \\
9 & 20 & 81662152 & 625218940868432 \\
\hline
\end{tabular}




\section{I. NiCOLAESCU}

In the main recurrence formula (5.1) we make the change in variables, $m \rightarrow m+1$. We deduce that for $n \geqslant m \geqslant 1$ we have the equality

$$
\begin{aligned}
G(m, n)-G(m+1, n)= & \left(\begin{array}{c}
m+1 \\
2
\end{array}\right) G(m-1, n-1) \\
& +\frac{m}{2} \sum_{n_{1}+n_{2}=n-1} \sum_{m_{1}+m_{2}=m}\left(\begin{array}{c}
m \\
m_{1}
\end{array}\right)\left(\begin{array}{c}
2 n-m \\
2 n_{1}-m_{1}+1
\end{array}\right) G\left(m_{1}, n_{1}\right) G\left(m_{2}, n_{2}\right) .
\end{aligned}
$$

Now we make the change in variables

$$
(m, n)=(x, x+y) \Longleftrightarrow(x, y)=(m, n-m), \quad H(x, y):=G(m, n) .
$$

With this notation the number of geometric equivalence classes of Morse functions with $2 n+2$ critical points is

$$
F_{n}(1)=G(0, n)=H(0, n)
$$

Then we have

$$
G(m+1, n)=H(x+1, y-1), \quad G(m-1, n-1)=H(x-1, y) .
$$

For $k=1,2$ we make the change in variables in the double sum

$$
\left(m_{k}, n_{k}\right)=\left(x_{k}, x_{k}+y_{k}\right) \Longleftrightarrow\left(x_{k}, y_{k}\right)=\left(m_{k}, n_{k}-m_{k}\right) .
$$

Then

$$
x_{2}=m-x_{1}=x-x_{1}, \quad y_{2}=n-1-y_{1}=x+y-1-y_{1},
$$

so that the equality

$$
\left(m_{1}, n_{1}\right)+\left(m_{2}, n_{2}\right)=(m, n-1)
$$

is equivalent to

$$
\left(x_{1}, x_{1}+y_{1}\right)+\left(x_{2}, x_{2}+y_{2}\right)=(x, x+y-1) \text {, }
$$

which implies that

$$
x_{1}+x_{2}=x, \quad y_{1}+y_{2}=y-1 .
$$

Now observe that in the double sum we need to have

$$
\begin{gathered}
2 n_{1}-m_{1}+1 \leqslant 2 n-m \Longrightarrow 2 x_{1}+y_{1}+1 \leqslant 2 x+y, \\
2 n_{2}-m_{2}+1 \leqslant 2 n-m \Longleftrightarrow 2\left(x-x_{1}\right)+\left(y-1-y_{1}\right)+1 \leqslant 2 x+y \Longleftrightarrow 0 \leqslant 2 x_{1}+y_{1} .
\end{gathered}
$$

These inequalities are satisfied if and only if

$$
\left(x_{1}, y_{1}\right) \in R_{x, y-1}:=\left\{(u, v) \in \mathbb{Z}^{2} ; 0 \leqslant u \leqslant x, 0 \leqslant v \leqslant y-1\right\} .
$$

For a point $\left(x_{1}, y_{1}\right) \in R_{x, y-1}$ we denote by $\left(\bar{x}_{1}, \bar{y}_{1}\right)$ its reflection in the center of $R_{x, y-1}$, that is,

$$
\left(x_{1}, y_{1}\right)+\left(\bar{x}_{1}, \bar{y}_{1}\right)=(x, y-1) .
$$

The recurrence can now be rewritten as

$$
\begin{aligned}
H(x, y)-H(x+1, y-1)= & \left(\begin{array}{c}
x+1 \\
2
\end{array}\right) H(x-1, y) \\
& +\frac{x+1}{2} \sum_{\left(x_{1}, y_{1}\right) \in R_{x, y-1}}\left(\begin{array}{c}
x \\
x_{1}
\end{array}\right)\left(\begin{array}{c}
x+2 y \\
x_{1}+2 y_{1}+1
\end{array}\right) H\left(x_{1}, y_{1}\right) H\left(\bar{x}_{1}, \bar{y}_{1}\right) .
\end{aligned}
$$

We now introduce the new function

$$
\hat{H}(x, y):=\frac{1}{x !(x+2 y+1) !} H(x, y) .
$$

Observe that $\hat{H}(0,0)=H(0,0)=G(0,0)=1$. We distinguish two cases. 
$A: x>0$. If we divide both sides of (6.2) by $x !(x+2 y)$ ! we deduce that for $x>0$ we have

$$
\begin{aligned}
(x & +2 y+1) \hat{H}(x, y)-(x+1) \hat{H}(x+1, y-1) \\
& =\frac{x+1}{2} \hat{H}(x-1, y)+\frac{x+1}{2} \sum_{\left(x_{1}, y_{1}\right) \in R_{x, y-1}} \hat{H}\left(x_{1}, y_{1}\right) \hat{H}\left(\bar{x}_{1}, \bar{y}_{1}\right) .
\end{aligned}
$$

Observe that if in the above equality we let $y=0$ we conclude

$$
\hat{H}(x, 0)=\frac{1}{2} \hat{H}(x-1,0) \text { so that } \hat{H}(x, 0)=2^{-x} \hat{H}(0,0)=2^{-x} .
$$

$B: x=0$. If we divide both sides of $(6.2)$ by $(2 y)$ ! we obtain

$$
(2 y+1) \hat{H}(0, y)-\hat{H}(1, y-1)=\frac{1}{2} \sum_{y_{1}=0}^{y-1} \hat{H}\left(0, y_{1}\right) \hat{H}\left(0, y-1-y_{1}\right) .
$$

Consider the formal power series

$$
\mathcal{H}(s, t):=\sum_{x, y \geqslant 0} \hat{H}(x, y) s^{x} t^{y}
$$

If we multiply both sides of cases $\mathrm{A}$ and $\mathrm{B}$ by $s^{x} t^{y-1}$ and sum over $x \geqslant 0, y \geqslant 1$ we deduce

$$
\begin{aligned}
& \sum_{x \geqslant 0, y \geqslant 1}(x+2 y+1) \hat{H}(x, y) s^{x} t^{y-1}-\sum_{x \geqslant 0, y \geqslant 1}(x+1) \hat{H}(x+1, y-1) s^{x} t^{y-1} \\
& =\sum_{x \geqslant 1, y \geqslant 1} \frac{x+1}{2} \hat{H}(x-1, y) s^{x} t^{y-1}+\sum_{x \geqslant 0, y \geqslant 1} \frac{x+1}{2}\left(\sum_{R_{x, y-1}} \hat{H}\left(x_{1}, y_{1}\right) \hat{H}\left(\bar{x}_{1}, \bar{y}_{1}\right)\right) s^{x} t^{y-1} .
\end{aligned}
$$

Make the change in variables $y=y+1$. Then

$$
\begin{aligned}
& \sum_{x \geqslant 0, y \geqslant 0}(x+2 y+3) \hat{H}(x, y+1) s^{x} t^{y}-\sum_{x \geqslant 0, y \geqslant 0}(x+1) \hat{H}(x+1, y) s^{x} t^{y} \\
& =\sum_{x \geqslant 1, y \geqslant 0} \frac{x+1}{2} \hat{H}(x-1, y+1) s^{x} t^{y}+\sum_{x \geqslant 0, y \geqslant 0} \frac{x+1}{2}\left(\sum_{R_{x, y}} \hat{H}\left(x_{1}, y_{1}\right) \hat{H}\left(\bar{x}_{1}, \bar{y}_{1}\right)\right) s^{x} t^{y} .
\end{aligned}
$$

Now make the change in variables $x=x+1$ in the third sum:

$$
\begin{aligned}
& \sum_{x \geqslant 0, y \geqslant 0}(x+2 y+3) \hat{H}(x, y+1) s^{x} t^{y}-\sum_{x \geqslant 0, y \geqslant 0}(x+1) \hat{H}(x+1, y) s^{x} t^{y} \\
& =\sum_{x \geqslant 0, y \geqslant 0} \frac{x+2}{2} \hat{H}(x, y+1) s^{x+1} t^{y}+\sum_{x \geqslant 0, y \geqslant 0} \frac{x+1}{2}\left(\sum_{R_{x, y}} \hat{H}\left(x_{1}, y_{1}\right) \hat{H}\left(\bar{x}_{1}, \bar{y}_{1}\right)\right) s^{x} t^{y} .
\end{aligned}
$$

We obtain

$$
\frac{1}{t} \partial_{s}\left(s \mathcal{H}-s \mathcal{H}_{t=0}\right)+2 \partial_{t} \mathcal{H}-\partial_{s} \mathcal{H}=\frac{1}{2 t} \partial_{s}\left(s^{2} \mathcal{H}-s^{2} \mathcal{H}_{t=0}\right)+\frac{1}{2} \partial_{s}\left(s \mathcal{H}^{2}\right) .
$$

From the equality

$$
\mathcal{H}_{t=0}=\mathcal{H}(s, 0)=\sum_{x \geqslant 0} 2^{-x} s^{x}=\frac{2}{2-s}
$$

we obtain

$$
\frac{1}{t} \partial_{s}(s \mathcal{H})+2 \partial_{t} \mathcal{H}-\partial_{s} \mathcal{H}=\frac{1}{2} \partial_{s}\left(s \mathcal{H}^{2}\right)+\frac{1}{2 t} \partial_{s}\left(s^{2} \mathcal{H}\right)+\frac{1}{t} \partial_{s} \frac{2 s-s^{2}}{2-s} .
$$

Multiplying both sides by $t$ we obtain

$$
\partial_{s}(s \mathcal{H})+2 t \partial_{t} \mathcal{H}-t \partial_{s} \mathcal{H}=\frac{t}{2} \partial_{s}\left(s \mathcal{H}^{2}\right)+\frac{1}{2} \partial_{s}\left(s^{2} \mathcal{H}\right)+1, \quad \mathcal{H}(s, 0)=\frac{2}{2-s} .
$$




\section{I. NicOlAescu}

The above equality is a first-order quasilinear PDE. However, the initial condition is characteristic (see [CH89, §II.1]), and thus the method of characteristics is ineffective in this case. To remove the singularities of this equation we blow it up via a monoidal change of coordinates

$$
s:=u v, \quad t:=v^{2}, \quad \xi(u, v):=v \mathcal{H}\left(u v, v^{2}\right) .
$$

Note that

$$
\xi(u, v)=\sum_{x, y \geqslant 0} H(x, y) \frac{u^{x} v^{x+2 y+1}}{x !(x+2 y+1) !}=\sum_{n+1 \geqslant m \geqslant 1} F_{n}(m) \frac{u^{m-1} v^{(2 n-m+2)}}{(m-1) ! \cdot(2 n-m+2) !},
$$

and

$$
\xi(0, v)=\sum_{n \geqslant 0} F_{n}(1) \frac{v^{2 n+1}}{(2 n+1) !},
$$

so that $\xi(0, v)$ is an exponential generating function for the numbers of geometric equivalence classes of excellent Morse functions on $S^{2}$. We have

$$
\begin{gathered}
v=t^{1 / 2}, \quad u=s t^{-1 / 2} \\
\mathcal{H}=v^{-1} \xi, \quad s \mathcal{H}=u \xi, \quad s^{2} \mathcal{H}=u^{2} v \xi, \quad s \mathcal{H}^{2}=u v^{-1} \xi^{2}, \\
\partial_{s}=\left(\partial_{s} u\right) \partial_{u}+\left(\partial_{s} v\right) \partial_{v}=t^{-1 / 2} \partial_{u}=v^{-1} \partial_{u}, \\
\partial_{t}=\left(\partial_{t} u\right) \partial_{u}+\left(\partial_{t} v\right) \partial_{v}=-\frac{s t^{-3 / 2}}{2} \partial_{u}+\frac{t^{1 / 2}}{2} \partial_{v}=\frac{1}{2 v^{2}}\left(-u \partial_{u}+v \partial_{v}\right) .
\end{gathered}
$$

Equation (6.4) can now be rewritten as

$$
v^{-1} \partial_{u}(u \xi)+\left(-u \partial_{u}+v \partial_{v}\right)\left(v^{-1} \xi\right)-v \partial_{u}\left(v^{-1} \xi\right)=\frac{1}{2} \partial_{u}\left(u \xi^{2}\right)+\frac{1}{2} \partial_{u}\left(u^{2} \xi\right)+1 .
$$

After some elementary manipulations we obtain the first-order quasilinear PDE

$$
-\left(1+u \xi+\frac{1}{2} u^{2}\right) \partial_{u} \xi+\partial_{v} \xi=\frac{1}{2} \xi^{2}+u \xi+1, \quad \xi(u, 0)=0
$$

The characteristic vector field of this equation is (see $[$ Arn88, $\S 7 . \mathrm{E}]$ or [CH89, II.1]) the vector field $\mathbf{V}$ in the three-dimensional vector space with coordinates $(u, v, \xi)$ defined by

$$
\mathbf{V}=-\left(1+u \xi+\frac{1}{2} u^{2}\right) \partial_{u}+\partial_{v}+\left(1+u \xi+\frac{1}{2} \xi^{2}\right) \partial_{\xi}
$$

Consider the curve $\sigma \rightarrow \gamma(\sigma)$ in the $(u, v, \xi)$-space described by the initial conditions

$$
u=\sigma, \quad v=0, \quad \xi=\xi(\sigma, 0)=0 .
$$

The tangent vector field $d \gamma / d \sigma$ coincides with the vector field $\partial_{u}$. Along $\gamma(\sigma)$ we have $\mathbf{V}(\sigma, 0,0)=$ $-\left(1+\sigma^{2} / 2\right) \partial_{u}+\partial_{v}+\partial_{\xi}$ which, together with the equality $d \gamma / d \sigma=\partial_{u}$, shows that the initial curve is non-characteristic.

The characteristic curves of (6.5) are the integral curves of $\mathbf{V}$, that is, the solutions of the system of ordinary differential equations

$$
\left\{\begin{array}{l}
\frac{d u}{d \tau}=-\left(1+u \xi+\frac{u^{2}}{2}\right), \\
\frac{d v}{d \tau}=1 \\
\frac{d \xi}{d \tau}=1+u \xi+\frac{1}{2} \xi^{2} .
\end{array}\right.
$$

The graph of the function $(u, v) \mapsto \xi(u, v)$ is filled in a neighborhood of $(u, v)=(0,0)$ by the solutions of (6.6) with initial points on $\gamma$. More precisely, if

$$
\tau \longmapsto\left(u_{\sigma}(\tau), v_{\sigma}(\tau), \xi_{\sigma}(\tau)\right)
$$




\section{MORSE FUnCTIONS ON THE 2-SPHERE}

is the solution of (6.6) satisfying the initial condition

$$
u_{\sigma}(0)=\sigma, \quad v_{\sigma}(0)=0, \quad \xi_{\sigma}(0)=0,
$$

then the graph of $\xi$ admits the parametric description

$$
(\sigma, \tau) \longmapsto\left(u_{\sigma}(\tau), v_{\sigma}(\tau), \xi_{\sigma}(\tau)\right) .
$$

We deduce that $v_{\sigma}(\tau)=\tau$, and that the plane curve $\tau \mapsto\left(u_{\sigma}(\tau), \xi_{\sigma}(\tau)\right)$ is a solution of the hamiltonian equation

$$
\left\{\begin{array}{l}
\frac{d u_{\sigma}}{d \tau}=-\partial_{\xi} h\left(u_{\sigma}, \xi_{\sigma}\right), \\
\frac{d \xi_{\sigma}}{d \tau}=\partial_{u} h\left(u_{\sigma}, \xi_{\sigma}\right),
\end{array}\right.
$$

where

$$
h(u, \xi):=\frac{1}{2}\left(u^{2} \xi+u \xi^{2}\right)+u+\xi=(u+\xi)\left(\frac{1}{2} u \xi+1\right) .
$$

We deduce that $h(u, \xi)=$ constant along the trajectories of (6.6). Thus, the solutions of the initial value problem $(6.6)+(6.7)$ satisfy

$$
h\left(u_{\sigma}(\tau), \xi_{\sigma}(\tau)\right)=h\left(u_{\sigma}(0), \xi_{\sigma}(0)\right) \text { for all } \tau,
$$

that is,

$$
\frac{1}{2}\left(u_{\sigma}^{2} \xi_{\sigma}+u_{\sigma} \xi_{\sigma}^{2}\right)+u_{\sigma}+\xi_{\sigma}=\sigma .
$$

We interpret this equality as a quadratic equation in $\xi_{\sigma}$

$$
\frac{u_{\sigma}}{2} \xi_{\sigma}^{2}+\left(1+\frac{u_{\sigma}^{2}}{2}\right) \xi_{\sigma}+u_{\sigma}-\sigma=0
$$

and we solve for $\xi_{\sigma}$

$$
\xi_{\sigma}=\frac{-\left(1+u_{\sigma}^{2} / 2\right)+\sqrt{\left(1+u_{\sigma}^{2} / 2\right)^{2}-2 u_{\sigma}\left(u_{\sigma}-\sigma\right)}}{u_{\sigma}} .
$$

In the above, the choice of a plus sign in the quadratic formula is dictated by the fact that the Taylor coefficients of $\xi$ (as a function of $u$ and $v$ ) are positive. Thus,

$$
1+u_{\sigma} \xi_{\sigma}+\frac{u_{\sigma}^{2}}{2}=\sqrt{\left(1+\frac{u_{\sigma}^{2}}{2}\right)^{2}-2 u_{\sigma}\left(u_{\sigma}-\sigma\right)}=\sqrt{\frac{u_{\sigma}^{4}}{4}-u_{\sigma}^{2}+2 \sigma u_{\sigma}+1}
$$

and using the first equation in (6.6) we deduce that

$$
\frac{d u_{\sigma}}{d \tau}=-\sqrt{\frac{u_{\sigma}^{4}}{4}-u_{\sigma}^{2}+2 \sigma u_{\sigma}+1}
$$

Set

$$
P_{\sigma}(u):=\frac{u^{4}}{4}-u^{2}+2 \sigma u+1, \quad \theta=\theta_{\sigma}(u):=\int_{0}^{u} \frac{d x}{\sqrt{P_{s}(x)}} .
$$

For fixed $\sigma$ we regard the time variable $\tau$ as a function ${ }^{3}$ of $u_{\sigma}$. We deduce from (6.9) that

$$
\tau=C_{\sigma}-\theta_{\sigma}\left(u_{\sigma}\right)
$$

where the constant $C_{\sigma}$ is determined from the initial condition

$$
\tau=0, \quad u_{\sigma}=\sigma .
$$

\footnotetext{
${ }^{3}$ For fixed $\sigma$, the inverse function $u_{\sigma} \mapsto \tau$ is an elliptic function.
} 


\section{I. NiCOLAESCU}

Hence,

$$
C_{\sigma}=\theta_{\sigma}(\sigma)=\int_{0}^{\sigma} \frac{d x}{\sqrt{P_{\sigma}(x)}}
$$

The moment of time $\tau(\sigma)$ when the characteristic curve

$$
\tau \longmapsto\left(u_{\sigma}(\tau), v_{\sigma}(\tau), \xi_{\sigma}(\tau)\right)
$$

(in the space $\mathbb{R}^{3}$ with coordinates $(u, v, \xi)$ ) intersects the hyperplane $u=0$ is obtained from the equality

$$
\tau(\sigma)=C_{\sigma}-\theta_{\sigma}\left(u_{\sigma}=0\right)=C_{\sigma}=\theta_{\sigma}(\sigma) .
$$

We deduce that the intersection of the hyperplane $u=0$ with the graph of $\xi$ admits the parametrization

$$
\sigma \longmapsto\left(0, \tau(\sigma), \xi_{\sigma}(\tau(\sigma))\right)
$$

From (6.8) we deduce that when $u_{\sigma}=0$ we have $\xi_{\sigma}(\tau(\sigma))=\sigma$, so that the intersection of the graph of $\xi$ with the hyperplane $u=0$ admits the parametrization

$$
\sigma \mapsto(0, \tau(\sigma), \sigma)
$$

On the other hand, if we think of $\xi$ as a function of variables $(u, v)$, the intersection of the graph of $\xi$ with the hyperplane $u=0$ admits the parametrization

$$
v \mapsto(0, v, \xi(0, v)) .
$$

The last two equalities show that

$$
\xi(0, \tau(\sigma))=\sigma, \quad \text { where } \tau(\sigma)=\int_{0}^{\sigma} \frac{d x}{\sqrt{P_{\sigma}(x)}} .
$$

We have thus proved the following result.

Theorem 6.1. Denote by $g_{n}$ the number of geometric equivalence classes of Morse functions on $S^{2}$ with $n$ saddle points and set

$$
\xi(t)=\sum_{n \geqslant 0} g(n) \frac{t^{2 n+1}}{(2 n+1) !}
$$

Then $\xi(t)$ is the compositional inverse of the function

$$
\theta(s)=\int_{0}^{s} \frac{d \tau}{\sqrt{\tau^{4} / 4-\tau^{2}+2 s \tau+1}} \stackrel{(\tau=s x)}{=} s \int_{0}^{1} \frac{d x}{\sqrt{\frac{1}{4}(s x)^{4}-(s x)^{2}+2 s^{2} x+1}},
$$

that is, $\xi(\theta(s))=s$.

Remark 6.2. (a) We can produce an explicit parametrization of the graph of $\xi$. Since $u$ is the solution of the initial value problem

we deduce that

$$
\frac{d u}{d \tau}=-\sqrt{P_{\sigma}(\tau)}, \quad u(0)=\sigma
$$

$$
-\tau=\int_{\sigma}^{u} \frac{d x}{\sqrt{P_{\sigma}(x)}} .
$$

Using the Weierstrass formula [WW27, §20.6, Example 2] we obtain the identity

$$
u=\sigma+\frac{P_{\sigma}(\sigma)^{1 / 2} \wp_{\sigma}^{\prime}(-\tau)+\frac{1}{2} P_{\sigma}^{\prime}(\sigma)\left(\wp_{\sigma}(-\tau)-\frac{1}{24} P_{\sigma}^{(2)}(\sigma)\right)+\frac{1}{24} P_{\sigma}(\sigma) P_{\sigma}^{(3)}(\sigma)}{2\left(\wp_{\sigma}(-\tau)-\frac{1}{24} P_{\sigma}^{(2)}(\sigma)\right)-\frac{1}{48} P_{\sigma}(\sigma) P_{\sigma}^{(4)}(\sigma)},
$$


where $\wp_{\sigma}(z)$ is the Weierstrass function with parameters

$$
g_{2}=g_{2}(\sigma)=\frac{1}{3}, \quad g_{3}=g_{3}(\sigma)=-\frac{1}{27}-\frac{\sigma^{2}}{16} .
$$

The discriminant is

$$
\Delta(\sigma)=g_{2}^{3}-27 g_{3}^{2}=-\frac{\sigma^{2}}{8}\left(1+\frac{27}{32} \sigma^{2}\right) .
$$

Using the equality $v=\tau$, and the description of $\xi$ as a function of $u$ and $\sigma$ we obtain a parametrization of the graph of $\xi$ in a small neighborhood of the point $(u, v, \xi)=(0,0,0)$.

Example 6.3. The Taylor coefficients of $\xi(t)$ can, in principle, be computed from the formula in Theorem 6.1 via the Lagrange inversion formula although this procedure is not as effective as the recurrence in Theorem 5.1. However, we want to test the validity of Theorem 6.1 on special cases.

For every formal power series $f$ in the variable $x$ we denote by $\left[x^{n}\right] f$ the coefficient of $x^{n}$ in the expansion of $f$. The Lagrange inversion formula [Sta99, Theorem 5.4.2] implies that

$$
\left[t^{5}\right] \xi=\frac{1}{5}\left[s^{4}\right]\left(\frac{s}{\theta(s)}\right)^{5} .
$$

We write

$$
P_{s}(t)=1+r_{s}(t), \quad r_{s}(t)=2 s t-t^{2}+\frac{t^{4}}{4}
$$

Then we have a binomial expansion

$$
P_{s}(t)^{-1 / 2}=1-\frac{1}{2} r_{s}(t)+\frac{1 \cdot 3}{2^{2} \cdot 2 !} r_{s}(t)^{2}-\frac{1 \cdot 3 \cdot 5}{2^{3} \cdot 3 !} r_{s}(t)^{3}+\frac{1 \cdot 3 \cdot 5 \cdot 7}{2^{4} \cdot 4 !} r_{s}(t)^{4}+\cdots .
$$

Integrating this equality with respect to $t \in[0, s]$ we deduce that

$$
\theta(s)=s \int_{0}^{1} P_{s}(s x)^{-1 / 2} d x=s(1-\underbrace{\left(\frac{1}{3} s^{2}-\frac{7}{40} s^{4}+\frac{3}{28} s^{6}+\cdots\right)}_{=: q(s)}) .
$$

Then

and we deduce that

$$
\frac{s}{\theta(s)}=(1-q(s))^{-1}
$$

$$
\left[s^{4}\right]\left(\frac{s}{\theta(s)}\right)^{5}=5\left[s^{4}\right] q(s)+15\left[s^{4}\right] q(s)^{2}=-\frac{35}{40}+\frac{15}{9}=\frac{19}{24} \Longrightarrow 5 !\left[t^{5}\right] \xi=19 .
$$

This agrees with the value computed in Table 1. Similarly,

$$
\left[s^{6}\right]\left(\frac{s}{\theta(s)}\right)^{7}=7\left[s^{6}\right] q(s)+\frac{7 \cdot 8}{2 !}\left[s^{6}\right] q(s)^{2}+\frac{7 \cdot 8 \cdot 9}{3 !}\left[s^{6}\right] q(s)^{3}=\frac{107}{180}
$$

which shows that $7 !\left[t^{7}\right] \xi=428$. This too agrees with the value found in Table 1 .

\section{On the topological equivalence problem}

We were not able to find a computationally satisfactory recurrence for the number of topological equivalence classes of Morse functions but we could still describe some interesting combinatorial structures on this set.

Assume that we are given a slicing $a_{0}<a_{1}<\cdots<a_{2 n+2}$ of an excellent Morse function $f: S^{2} \rightarrow \mathbb{R}$. For every $i=1,2, \ldots, 2 n+1$ we set $X^{i}:=\left\{f \leqslant a_{i}\right\}$. We find it convenient to encode the topological transitions $X^{i} \rightarrow X^{i+1}$ as a game of plates and olives. 


\section{I. NicOlAESCU}
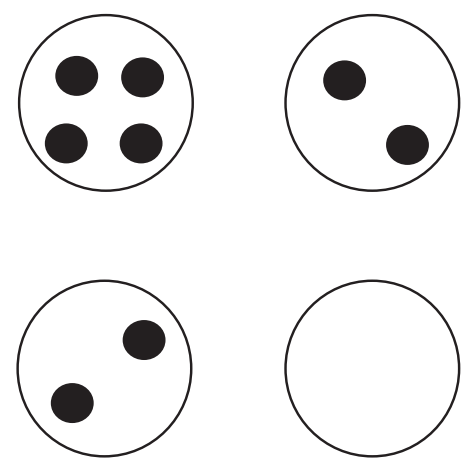

FIGURE 13. Black olives on white plates.

More precisely, every sublevel set $X^{i}$ is homeomorphic to a collection 2-disks with a number of holes in them. One can visualize a disk as a white plate, and a hole in a disk as a black olive on that plate (see Figure 13). The four possible types of handle attachments are encoded by the following four possible transformations of a distribution of identical plates and identical olives (on those plates).

- $H_{0}$. Add a new empty plate.

- $H_{1}^{+}$. Add a new olive on one of the existing plates.

- $H_{1}^{-}$. Remove an empty plate, or place all of the olives from one existing plate on another existing plate, and then remove the emptied plate.

- $\mathrm{H}_{2}$. Remove one of the existing olives.

We refer to the above four transformations as (admissible) moves. A game of plates and olives is a sequence of distributions of identical olives on identical plates, starting and ending with one empty plate, and such that any two consecutive distributions are related by one of the above four admissible moves. The length of a game is equal to the number of moves.

Arguing as in the proof of Lemma 4.1 we conclude that the number of topological equivalence classes of Morse functions on $S^{2}$ of order $n$ is equal to the number of plates-and-olives games of length $2 n$.

A distribution of plates and olives can be encoded by a partition $\pi$, that is, a nonincreasing function

$$
\pi: \mathbb{Z}_{>0} \rightarrow \mathbb{Z}_{\geqslant 0}, \quad \pi(i) \geqslant \pi(i+1), \quad \text { for all } i>0,
$$

such that $\pi(i)=0$ for all $i \gg 0$. The length of the partition $\pi$ is by definition the cardinality of its support. The weight of the partition is the integer

$$
|\pi|=\sum_{i>0} \pi(i)
$$

If $n=|\pi|$ we say that $\pi$ is a partition of $n$. We denote by $\mathcal{P}$ the set of all partitions of nonnegative weight and by $\mathcal{P}_{n}$ the set of partitions of weight $n$. The character $\mathbf{0}$ denotes the unique partition of weight 0 .

To a partition $\pi$ of length $\ell$ there corresponds a collection of $\ell$ plates, $\pi(1)-1$ olives on the first plate, $\pi(2)-1$ olives on the second plate etc. The weight of the partition is equal to the total number of plates and olives. This correspondence between partitions and distributions of identical plates and identical olives is obviously a bijection. For example, to the distribution of plates and olives in Figure 13 it corresponds the partition 5,3,3, 1 of length 4 and weight $5+3+3+1=12$. 
Note that the $H_{0}$ move increases the length and the weight by one, the $H_{1}^{+}$move increases the weight by one, but preserves the length, the $H_{1}^{-}$move decreases the weight and the length by one, while the $\mathrm{H}_{2}$ move decreases the weight by one, but preserves the length.

We introduce a simplified set of moves, which we describe in the language of plates and olives.

- Type $U$ (up) move. Add an empty plate, or an olive on an existing plate.

- Type $D$ (down) move. Remove an olive, or an empty plate.

- Type $X$ move. Place all of the olives from one existing, nonempty plate on another existing plate, and then remove the emptied plate.

We can use these moves to produce a directed graph with vertex set $\mathcal{P}$. The procedure is very simple. For every move $U, D, X$

$$
\mathcal{P} \ni \pi \rightarrow \pi^{\prime} \in \mathcal{P}
$$

draw an arrow directed from $\pi$ to $\pi^{\prime}$, labelled by the corresponding symbol, $U, D, X$. We denote by $\hat{\mathcal{P}}$ this graph. Then the number of topological equivalence classes of Morse functions with $2 n+2$ critical points is equal to the number of directed paths of length $2 n$ from the partition 1 to itself. Equivalently, it is the number of directed paths of length $2 n+2$ from $\mathbf{0}$ to itself. We denote this number by $T_{2 n+2}$.

Observe that if there exists an $U$-arrow $\pi \rightarrow \pi^{\prime}$, then there exists a $D$-arrow $\pi^{\prime} \rightarrow \pi$ in the opposite direction. We say that a path in $\mathcal{P}$ is simple if it consists only of the $U, D$ moves. Given $\pi, \pi^{\prime} \in \mathcal{P}$ we write $\pi \prec \pi^{\prime}$ if there exists a path from $\pi$ to $\pi^{\prime}$ consisting only of $U$-moves. Clearly $\prec$ is a partial order on $\mathcal{P}$.

On the set $\mathcal{P}$ we have another partial order $\subsetneq$, known as the Young ordering (see [Sta99, ch. 7]), where

$$
\pi \subsetneq \pi^{\prime} \Longleftrightarrow|\pi|<\left|\pi^{\prime}\right| \text { and } \pi(i) \leqslant \pi^{\prime}(i), \quad \text { for all } i>0 .
$$

It is easy to see that

$$
\pi \prec \pi^{\prime} \Longleftrightarrow \pi \subsetneq \pi^{\prime}
$$

We can now relate the number of simple paths of length $2 n+2$ from $\mathbf{0}$ and back, to walks in the Young lattice starting and ending at $\mathbf{0}$. The number of such walks is (see [Sta88, (39)])

$$
S_{2 n+2}=\frac{(2 n+2) !}{2^{n+1}(k+1) !}=(2 n+1) ! !=1 \cdot 3 \cdots(2 n+1) .
$$

In particular, we deduce the following result.

Proposition 7.1. The number $T_{2 n+2}$ of topological equivalence classes of Morse functions with $2 n+2$ critical points satisfies the lower bound

$$
T_{2 n+2} \geqslant(2 n+1) ! !
$$

Remark 7.2. (a) Since the $X$-moves do not affect partitions which have only one part greater than one we deduce that for $n=2$ we have $T_{6}=S_{6}=15$ so that there are exactly 15 topological equivalence classes of Morse functions on $S^{2}$ with six critical points.

(b) A simple diagram chasing coupled with the hook-length formula [Sta99, ch. 7] leads to the equalities $T_{8}=107, T_{10}=981$. The diagram chasing however becomes increasingly unmanageable for large $n$.

\section{ACKNOWLEDGEMENTS}

I would like to thank Professor V. I. Arnold for his kind interest, suggestions and comments concerning this work. I also want to thank the anonymous referee for his/her constructive suggestions. 


\section{MORSE FUNCTIONS ON THE 2-SPHERE}

\section{REFERENCES}

Arn88 V. I. Arnold, Geometrical methods in the theory of ordinary differential equations, second edition (Springer, Berlin, 1988).

Arn91 V. I. Arnold, Bernoulli-Euler updown numbers associated with function singularities, their combinatorics and arithmetics, Duke Math. J. 63 (1991), 537-555.

Arn92 V. I. Arnold, The calculus of snakes and the combinatorics of Bernoulli, Euler and Springer numbers of Coxeter groups, Russian Math. Surveys 47 (1992), 3-45.

Arn06 V. I. Arnold, Smooth functions statistics, Funct. Anal. Other Math. 1 (2006), 135-178.

Arn07 V. I. Arnold, Topological classification of Morse functions and generalizations of Hilbert's 16th problem, Math. Phys. Anal. Geom. 10 (2007), 227-236.

BF04 A. V. Bolsinov and A. T. Fomenko, Integrable Hamiltonian systems. Geometry, topology classification (Chapman and Hall/CRC, Boca Raton, FL, 2004).

CH89 R. Courant and D. Hilbert, Methods of mathematical physics, vol. II (John Wiley and Sons, New York, 1989).

GZ92 I. M. Gessel and D. Zeilberger, Random walk in a Weyl chamber, Proc. Amer. Math. Soc. 115 (1992), $27-31$.

Guy01 R. K. Guy, Catwalks, sandsteps and Pascal pyramids, J. Integer Seq. 3 (2001), http://www.cs.uwaterloo.ca/journals/JIS/VOL3/GUY/catwalks.html.

Kud99 E. A. Kudryavtseva, On the realization of smooth functions on a surface in the form of functions of height, Mat. Sb. 190 (1999), 29-88; Engl. transl. Sb. Math. 190 (1999), 349-405.

Kul98 E. V. Kulinich, On topologically equivalent of Morse functions on surfaces, Methods Funct. Anal. Topology 4 (1998), 59-64.

Mil65 J. W. Milnor, Lectures on the h-cobordism (Princeton University Press, Princeton, 1965).

Nic06 L. I. Nicolaescu, Morse functions statistics, Funct. Anal. Other Math. 1 (2006), 85-91.

Ree46 G. Reeb, Sur les points singuliers d'une forme de Pfaff complètement intégrable ou d'une fonction numérique, C. R. Math. Acad. Sci. Paris 222 (1946), 847-849.

Sha03 V. V. Sharko, Smooth and topological equivalence of functions on surfaces, Ukrainian Math. J. 55 (2003), 832-846.

Sta88 R. P. Stanley, Differentiable posets, J. Amer. Math. Soc. 1 (1988), 919-961.

Sta99 R. P. Stanley, Enumerative combinatorics, Vol. II, Cambridge Studies in Advanced Mathematics, vol. 62 (Cambridge University Press, Cambridge, 1999).

VW92 J. H. van Lint and R. M. Wilson, A course in combinatorics (Cambridge University Press, Cambridge, 1992).

WW27 E. T. Whittaker and G. N. Watson, A course of modern analysis (Cambridge University Press, Cambridge, 1996).

Liviu I. Nicolaescu nicolaescu.1@nd.edu

Department of Mathematics, University of Notre Dame, Notre Dame, IN 46556-4618, USA. 